\title{
Optical photometry of the UCM lists I and II
}

\section{B band surface photometry and morphological discussion ${ }^{\star}$}

\author{
P. G. Pérez-González, J. Gallego, J. Zamorano, and A. Gil de Paz
}

\begin{abstract}
Departamento de Astrofísica, Universidad Complutense de Madrid, Av. Complutense s/n. 28040 Madrid, Spain e-mail: pag@astrax.fis.ucm.es; jgm@astrax.fis.ucm.es; jaz@astrax.fis.ucm.es; gil@astrax.fis.ucm.es
\end{abstract}

Received 2 August 2000 / Accepted 16 October 2000

\begin{abstract}
We present Johnson $B$ surface photometry for the UCM Survey galaxies. One-dimensional bulge-disk decomposition is attempted, discussing on fitting functions and computational procedures. The results from this decomposition, jointly with concentration indices and an asymmetry coefficient, are employed to study the morphological properties of these galaxies. We also compare our results with the previous morphological classification established using Gunn $r$ imaging data and with other samples of galaxies. No major statistical differences in morphology are found between red and blue data, although some characteristics such as size and luminosity concentration vary. We find a correlation between luminosity and size. Several parameters are used to segregate the objects according to their morphological type.
\end{abstract}

Key words. galaxies: photometry - galaxies: fundamental parameters - surveys-galaxies-spiral, galaxies-starburst - methods: data analysis

\section{Introduction}

The Universidad Complutense de Madrid Survey (UCM Survey list I, Zamorano et al. 1994; list II, Zamorano et al. 1996; list III, Alonso et al. 1999) constitutes a representative and fairly complete sample of current star-forming galaxies in the Local Universe (Gallego 1999). Its main purposes are to identify and study new young, low metallicity galaxies and to quantify the properties of the current star formation in the Local Universe. Another key goal is also to provide a reference sample for the studies of highredshift populations, mainly dominated by star-forming galaxies (see, e.g., Hu et al. 1998; Steidel et al. 1999).

Specific details of the UCM sample concerning spectroscopic properties were presented in Gallego et al. (1995, 1996, 1997). Photometric properties in the Gunn $r$ band can be found in Vitores et al. (1996a, 1996b) and nearinfrarred data are available in Alonso-Herrero et al. (1996) and Gil de Paz et al. (2000).

In Pérez-González et al. (2000, hereafter Paper I), integrated Johnson $B$ photometry for the whole sample was presented. In this paper we will study the spatial concentration of the $B$ luminosity in these objects. This will

Send offprint requests to: P. G. Pérez-González

* Tables 2 and 3 are also available in electronic form at the CDS via anonymous ftp to

cdsarc.u-strasbg.fr (130.79.128.5) or via

http://cdsweb.u-strasbg.fr/cgi-bin/qcat?J/A+A/365/370 allow us to obtain information about morphology and the different structures embedded in the galaxies. The effects of the photometric band on the determination of galaxy morphology will be evaluated through the comparison of the results presented here with those achieved with the Gunn $r$ data (Vitores et al. 1996a, 1996b).

The study of large-scale characteristics of the galaxies involves the quantitative measurement of structural parameters and light distribution. These parameters, which must describe the different components of galaxies (i.e., bulges, disks, bars), may be used to perform a morphological classification of the objects.

In this sense, we will attempt bulge-disk decomposition in one dimensional surface photometry radial profiles. Calculation of concentration indices and an asymmetry coefficient will also be done. All these data will be utilized to classify the galaxies according to their Hubble type.

The paper is structured as follows: we introduce the sample of galaxies and the Johnson $B$ observations in Sect. 2. In Sect. 3 the method used in this surface photometry study is detailed, including the explanation of the procedures followed in the bulge-disk decomposition and in the calculation of concentration indices and the asymmetry coefficient. The results and discussion about morphology are located in Sect. 4. Finally, Sect. 5 presents the correlations found between several photometric parameters. A Hubble constant $H_{0}=50 \mathrm{~km} \mathrm{~s}^{-1} \mathrm{Mpc}^{-1}$ and 
a deceleration parameter $q_{0}=0.5$ have been used throughout this paper.

\section{The sample}

The present work refers to the 191 galaxies within the lists I and II of the UCM Survey. The main features of each galaxy as well as the observation parameters and reduction techniques were listed in Paper I.

The UCM Survey galaxies lie at an averaged redshift of 0.026 . The sample is dominated by low-excitation, high-metallicity starburst-like galaxies $(57 \%$ of the sample); high-excitation, low-metallicity HII-like galaxies are also present $(32 \%)$ with a fraction of AGN objects $(8 \%)$ (Gallego et al. 1996). The averaged $B$ magnitude and standard deviation of the sample are $16.1 \pm 1.1$ and the mean $B-r$ colour $0.73 \pm 0.41$ (Pérez-González et al. 2000), corresponding to a morphological type of Sbc, according to Fukugita et al. (1995).

\section{Data analysis}

\subsection{Surface photometry}

Surface photometry was performed with the ellipse task within the IRAF ${ }^{1}$ STSDAS.ISOPHOTE package.

The ellipse task matches elliptical isophotes to galaxy light intensity. The technique employed is described in Jedrzejewski (1987). It consists on an iterative method that fits ellipses to the intensity of the images at a given semi-major axis. Once the isophote is fitted, the semimajor axis is changed (increased or decreased). This task was run interactively always starting from the same semimajor axis (the one corresponding to 3 arcsec to avoid the part of the galaxy profile dominated by seeing). First, we proceeded outwards until we reached twice the Kron radius (Kron 1980) and then inwards.

Intensities were converted into surface brightnesses and plotted against the semi-major axes of the isophotes. No correction for inclination $(i)$ was attempted before fitting the profiles due to the great variety of uncertainties that the determination of $i$ involves. These surface brightness profiles, together with the bulge-disk adjustments explained in the Sect. 3.2, are available via anonymous ftp at the site 147.96.22.14.

Along with the surface brightness profiles, the method mentioned above also provides the ellipticity $\epsilon$ and position angle PA of each isophote. For each image, mean $\epsilon$ and PA were calculated with the values of the isophotes between 23 and $24 \mathrm{mag} \operatorname{arcsec}^{-2}$ and are listed in Table 2. Since the outer isophotes of many of our galaxies were distorted by different structures, such as bars, rings, spiral arms, bright HII regions, some of these averages were corrupted, so a visual inspection of each image was carried

\footnotetext{
${ }^{1}$ IRAF is distributed by the National Optical Astronomy Observatories, which is operated by the Association of Universities for Research in Astronomy, Inc. (AURA) under cooperative agreement with the National Science Foundation.
}

out in order to exclude from the averaging the distorted zones and get more indicative values.

\subsection{Bulge-disk decomposition. The method}

Traditionally, galaxy light distributions have been studied through the decomposition in distinct components (Kent 1985; de Jong 1996b; Vitores et al. 1996a; Baggett et al. 1998; among others). Most methods are based on the assumption of specific functions. Ideally, these functions should have a physical background, being connected with the formation and evolution of galaxies. Unfortunately this is a very hard task so authors commonly use empirically derived functions.

Light distributions of spiral galaxies are commonly modeled using two components: a central concentration of luminosity (the bulge) and an outer plane structure (the disk). This simple scheme can be far from the real component mixture of the galaxy. Features such as bars, rings or bright starbursts affect dramatically the light distribution and make bulge-disk decomposition a nearly impossible task. These features are supposed to be more frequent in late Hubble type galaxies and extremely relevant in starburst galaxies, becoming dominant at high-redshifts.

Bulge-disk decomposition can be undertaken using several techniques and fitting functions. Several authors are now using the entire galaxy image to perform two dimensional fittings of the flux (see, e.g., de Jong 1996b); this technique is better for galaxies with peculiar structures such as bars or rings, which are masqueraded in the azimuthally averaged plots.

We have carried out the morphology study of the sample using one-dimensional surface brightness profiles. These profiles were checked visually in order to exclude from the fitting algorithm those regions dominated by artifacts, which are revealed through bumps and dips in the radial profiles. Besides, the algorithm only utilizes the points with $\mu$ lower than the detection threshold, which was measured as the surface brightness corresponding to the standard deviation of the sky; the values of this threshold ranged from 24 to $26 \mathrm{mag} \operatorname{arcsec}^{-2}$, depending on the observation campaign. Some of the galaxies showed very irregular morphologies and extremely perturbed profiles due to interaction companions or starbursts; consequently, these galaxies were excluded from this bulge-disk study.

A great variety of fitting functions are available in the literature. Some authors adjust exponential laws to both bulge and disk or other more complicated functions. We have attempted the decompositions using the empirical bulge law established by de Vaucouleurs (1948):

$\mu=\mu_{\mathrm{e}}+8.33 \times\left(\left(\frac{r}{r_{\mathrm{e}}}\right)^{1 / 4}-1\right)$

and the classical exponential law for the disk (Freeman 1970):

$\mu=\mu_{0}+1.09 \times\left(\frac{r}{d_{\mathrm{L}}}\right)$ 
where $\mu$ stands for the surface brightness, $r$ for the radius, $r_{\mathrm{e}}$ for the bulge effective radius (that containing inside half of the total light of the bulge component), $\mu_{\mathrm{e}}$ the bulge effective surface brightness, $d_{\mathrm{L}}$ and $\mu_{0}$ the disk scale length and central surface brightness.

The choice of the classical $r^{1 / 4}$ and exponential fitting functions allows us to compare our results with the Gunn $r$ study and with most of the data found in the literature.

During the performance of bulge-disk decomposition, special care should be taken when dealing with the inner parts of the galaxy profile, since these zones are affected by atmospheric seeing. Most authors exclude from the fit the part of the galaxy dominated by seeing (e.g., Baggett et al. 1998; Schombert \& Bothun 1987; Chatzichristou 1999). To account for this effect, we used in the fitting procedure a seeing-convolved formula for the light profile in the inner parts of the galaxy (Pritchet \& Kline 1981). This procedure copes with the uncomfortable $r^{1 / 4}$ bulge law, that tends to infinity as $r$ approaches 0 . Assuming radial symmetry and a Gaussian description of the PSF, the seeing convolved profile can be expressed as:

$I_{\mathrm{c}}(r)=\sigma^{-2} \mathrm{e}^{-r^{2} / 2 \sigma^{2}} \int_{0}^{\infty} I(x) I_{0}\left(x r / \sigma^{2}\right) \mathrm{e}^{-x^{2} / 2 \sigma^{2}} x \mathrm{~d} x$

where $I_{\mathrm{c}}(r)$ is the seeing-convolved intensity, $\sigma$ the dispersion of the seeing Gaussian PSF, $I(x)$ the sum of the bulge and disk intensities and $I_{0}$ the zero-order modified Bessel function of the first kind. Seeing dispersions were measured on several field stars for each image; the averaged

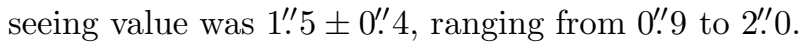

The main problem involved with seeing is the determination of the seeing-dominated zone of the profile, where Eq. (3) has to be used. This parameter was set free until a best fit was achieved.

The decomposition procedure followed to obtain the bulge and disk parameters is the following:

- All the fitting subroutines need a first guess for the bulge and disk parameters $\mu_{\mathrm{e}}, r_{\mathrm{e}}, \mu_{0}$ and $d_{\mathrm{L}}$. To calculate them we separated the bulge and disk dominated regions; the first one should be located in the centroid of the galaxy (with the most central zones dominated by seeing) and should be linear in a surface brightness versus $r^{1 / 4}$ plot; the zone dominated by the disk must be in the outer parts of the galaxy and should be linear in a $\mu$ versus $r$ plot. This decomposition provided a first estimate for the bulge and disk parameters;

- The next step is to fit the two components simultaneously. This was done using a $\chi^{2}$ minimization performed with the simplex method. Before this minimization, we proved several solutions in the parameter space around the data acquired in the first step in order to avoid local minima of the $\chi^{2}$ function. The simplex algorithm needs five sets of initial parameters that were built with the best of the previous values, varying them randomly;

- One final step was performed in order to calculate the best set of fitting parameters and their corresponding errors. Each data value in the surface brightness profile was varied randomly according to a Gaussian distribution; the sigma of this Gaussian was the standard deviation of the point calculated with the ellipse task. Each new profile was refitted using the simplex method. This process was repeated 1000 times. Then those fits with values of the function $\chi^{2}$ between the lowest value $\chi_{\min }^{2}$ and 3 times $\chi_{\min }^{2}$, were selected from the 1000 iterations. The final set of parameters were the averaged values of the latest and the errors corresponded to the standard deviations of these fits.

Equal weights were used for all the points during the fits. The outermost points of the profiles have larger errors due to the uncertainties in the determination of the sky, artifacts, etc. This should lead to assign greater weights to the innermost points, as some authors do in the literature (Baggett et al. 1998; Chatzichristou 1999; Hunt et al. 1999). However, in our profiles there were more points in the inner parts of the plots than in the outer ones; when weights were introduced in the fitting algorithm, wrong estimates of the parameters (the bulge parameters are the most affected ones) occurred; therefore, the equi-weighting scheme was chosen.

The method described above was tested in several artificial galaxies. They were built with known and representative bulge and disk parameters. We chose typical profiles for this test, including: (a) those with well-defined bulge and disk, (b) with a dominant disk, (c) with a dominant bulge, (d) a nearly linear profile (fitted with a disk by our method) and (e) a curved profile (identified as a bulge by our method). The artificial profiles were convolved with a common seeing value of 1 ." 5 ; the zone where this convolution was made was set randomly inside the typical interval of the true fits. Standard values of noise were added to the profile, based on real data. In Table 1 some of the input and output bulge and disk parameters are shown. The initial parameters seem to be well recovered by our technique; the largest differences correspond to profiles where the disk dominates although there is some contribution of a bulge component (test number 2, corresponding to a late-type spiral); these profiles were identified as an isolate disk by our method. Discrepancies were also present when one of the components is dominant (examples number 4 or 7, corresponding to a late-type spiral and an early-type galaxy, respectively); in this case, the parameters of the other component do not contribute much to the total profile and our method of decomposition does not recover the initial values (the errors of the $B / D$ ratio are specially affected and are not shown in the result table - they are substituted by three dots -), although this fact is irrelevant. We took special care with these types of profile during morphological classification based on bulge-disk decomposition.

One of the main problems during profile fitting is the fact that the hypersurface in the four parameters space $\left(\mu_{\mathrm{e}}, r_{\mathrm{e}}, \mu_{0}, d_{\mathrm{L}}\right)$ has many local minima. The minimization method must be able to determine the real absolute minimum, whose parameters must have physical 
Table 1. Bulge-disk decomposition test data

\begin{tabular}{clllll}
\hline $\begin{array}{c}\text { Profile type } \\
(1)\end{array}$ & $\begin{array}{c}\mu_{\mathrm{e}} \\
(2)\end{array}$ & $\begin{array}{c}r_{\mathrm{e}} \\
(3)\end{array}$ & $\begin{array}{c}\mu_{0} \\
(4)\end{array}$ & $\begin{array}{l}d_{\mathrm{L}} \\
(5)\end{array}$ & $\begin{array}{l}B / D \\
(6)\end{array}$ \\
\hline \hline $\mathrm{a}$ & 19.50 & 0.90 & 21.60 & 5.50 & 0.67 \\
& $19.47 \pm 0.77$ & $0.88 \pm 0.58$ & $21.53 \pm 0.81$ & $5.30 \pm 0.93$ & $0.66 \pm 0.36$ \\
\hline $\mathrm{b}$ & 26.00 & 13.80 & 21.50 & 4.60 & 0.51 \\
& $25.34 \pm 0.23$ & $5.95 \pm 4.17$ & $21.32 \pm 0.41$ & $4.54 \pm 1.42$ & $0.15 \pm 0.33$ \\
\hline $\mathrm{c}$ & 23.30 & 5.30 & 21.80 & 6.90 & 0.54 \\
& $23.30 \pm 0.34$ & $5.31 \pm 1.82$ & $21.84 \pm 0.62$ & $7.10 \pm 3.30$ & $0.52 \pm 0.27$ \\
\hline $\mathrm{d}$ & 31.20 & 4.60 & 18.70 & 1.30 & 0.00 \\
& $26.76 \pm 0.04$ & $1.60 \pm 0.99$ & $18.71 \pm 0.01$ & $1.30 \pm 0.02$ & $0.00 \pm 0.01$ \\
\hline $\mathrm{d}$ & 24.10 & 4.40 & 19.20 & 2.40 & 0.13 \\
& $23.30 \pm 0.48$ & $2.06 \pm 0.83$ & $19.16 \pm 0.04$ & $2.42 \pm 0.04$ & $0.05 \pm 0.02$ \\
\hline $\mathrm{e}$ & 20.80 & 3.40 & 21.60 & 6.80 & 1.88 \\
& $20.80 \pm 0.13$ & $3.40 \pm 0.39$ & $21.62 \pm 0.74$ & $6.94 \pm 2.81$ & $1.84 \pm 0.51$ \\
\hline $\mathrm{e}$ & 21.50 & 3.50 & 22.90 & 4.90 & 6.68 \\
& $21.55 \pm 0.16$ & $3.60 \pm 0.48$ & $23.13 \pm 4.90$ & $5.22 \pm 1.17$ & $7.35 \pm \ldots$ \\
\hline \hline
\end{tabular}

Results for the test of the bulge-disk decomposition procedure on seven artificial galaxies. Input parameters are in the first row and output results and their corresponding errors in the second one. Columns: (1) Profile type as explained in the text. (2) Effective surface brightness of the bulge in mag arcsec ${ }^{-2}$. (3) Effective radius of the bulge in arcsec. (4) Typical surface brightness of the disk in mag $\operatorname{arcsec}^{-2}$. (5) Exponential scale of the disk in arcsec. (6) Bulge-to-disk ratio.

meaning. To achieve this, all the initial parameters were varied randomly before attempting the fit; we also used several fractional convergence tolerances in each individual fit and boundaries on each parameter were taken in order to avoid solutions with no physical meaning.

With the four parameters of the disk-bulge decomposition, the bulge-to-disk luminosity ratio was calculated as follows:

$\frac{B}{D}=\frac{L_{B}}{L_{D}}=3.607\left(\frac{r_{\mathrm{e}}}{d_{\mathrm{L}}}\right)^{2} 10^{\left(-0.4\left(\mu_{\mathrm{e}}-\mu_{0}\right)\right)}$.

All the data referring to bulge-disk decomposition, along with mean ellipticities and position angles calculated as explained in Sect. 3.1, are shown in Table 2. Some galaxies were unsuitable to perform bulge-disk decomposition due to very perturbed profiles or bad-quality of the images. These galaxies have no data of the bulge and disk parameters. Other galaxies were fitted with only one component; bulge-to-disk ratios for these objects have very large errors and no physical meaning so they are not shown in Table 2. Position angles were only measured in those galaxies with ellipticities greater than 0.05 (for rounder isophotes, estimation of the PA is meaningless); two galaxies with $\epsilon=0.06$, but very large error values, have not PA measurement, either.

\subsection{Light concentration indices}

In order to characterize galaxies morphologically, we need a measure of the degree of concentration of light towards the central or the outermost regions of the object. Traditionally, this has been performed through the calculation of the bulge-to-disk ratio $B / D$ mentioned in the previous section. The methodology and degree of fidelity of the bulge-disk decomposition are trustful just for galaxy profiles where only these two components are present and no special features are found (Schombert \& Bothun 1987). As was already mentioned, some of our galaxies are dominated by bright features (mainly bars, spiral arms and bright starbursts) and it seems better to improve their morphological classification using concentration indices not based in previous component fits.

We have calculated for the whole sample three concentration indices in the same way that Vitores et al. (1996a) did for the Gunn $r$ bandpass:

- $c_{31}$, defined by de Vaucouleurs (1977) as the ratio between the radius containing $75 \%$ of the light of the galaxy $\left(r_{75}\right)$ and the radius with $25 \%$ of the galaxy luminosity $\left(r_{25}\right)$ :

$c_{31}=\frac{r_{75}}{r_{25}}$.

The calculation was performed integrating the flux of the galaxy in contiguous isophotes until 25 and $75 \%$ of the total flux were reached. The radii used in Eq. (5) are equivalent radii, calculated as the square root of the product of the semi-major and semi-minor axes. This index was found to correlate well with morphological type, decreasing from early to late-type galaxies (Gavazzi et al. 1990);

$-c_{42}$, defined by Kent (1985) as:

$c_{42}=5 \log \left(\frac{r_{80}}{r_{20}}\right)$

where $r_{80}$ and $r_{20}$ are the equivalent radii containing 80 and $20 \%$ of the total flux of the galaxy, respectively. These radii were calculated as the ones for $c_{31}$;

- $c_{\text {in }}(\alpha)$, as defined in Doi et al. (1993):

$c_{\mathrm{in}}(\alpha)=\frac{\int_{0}^{\alpha r\left(\mu_{\mathrm{L}}\right)} r I(r) \mathrm{d} r}{\int_{0}^{r\left(\mu_{\mathrm{L}}\right)} r I(r) \mathrm{d} r}$ 
Table 2. Bulge and disk parameters, bulge-to-disk ratio, ellipticity and position angle of the UCM Survey galaxies

\begin{tabular}{|c|c|c|c|c|c|c|c|}
\hline $\begin{array}{l}\text { UCM name } \\
(1)\end{array}$ & $\begin{array}{l}\mu_{\mathrm{e}} \\
(2) \\
\end{array}$ & $\begin{array}{l}r_{\mathrm{e}} \\
(3) \\
\end{array}$ & $\begin{array}{l}\mu_{0} \\
(4) \\
\end{array}$ & $\begin{array}{l}d_{\mathrm{L}} \\
(5) \\
\end{array}$ & $\begin{array}{c}B / D \\
(6) \\
\end{array}$ & $\begin{array}{c}\epsilon \\
(7) \\
\end{array}$ & $\begin{array}{r}\mathrm{PA} \\
(8) \\
\end{array}$ \\
\hline $0000+2140$ & $18.96 \pm 0.47$ & $0.69 \pm 0.70$ & $19.74 \pm 0.38$ & $4.15 \pm 0.68$ & $0.20 \pm 0.24$ & $0.28 \pm 0.01$ & $-52 \pm 4$ \\
\hline $0003+2200$ & $21.04 \pm 0.38$ & $0.07 \pm 0.56$ & $21.35 \pm 0.04$ & $4.73 \pm 0.40$ & $0.00 \pm 0.00$ & $0.59 \pm 0.04$ & $-80 \pm 2$ \\
\hline $0003+2215$ & $27.71 \pm 2.73$ & $21.19 \pm 4.72$ & $21.71 \pm 0.36$ & $6.04 \pm 2.60$ & $0.18 \pm 0.13$ & $0.59 \pm 0.04$ & $-2 \pm 3$ \\
\hline $0003+1955$ & - & - & - & - & - & $0.06 \pm 0.04$ & - \\
\hline $0005+1802$ & $21.31 \pm 0.35$ & $0.80 \pm 0.39$ & $20.57 \pm 0.09$ & $4.27 \pm 0.47$ & $0.06 \pm 0.03$ & $0.64 \pm 0.01$ & $76 \pm 1$ \\
\hline $0006+2332$ & $23.04 \pm 0.32$ & $2.38 \pm 1.12$ & $20.21 \pm 0.13$ & $6.52 \pm 0.85$ & $0.04 \pm 0.02$ & $0.58 \pm 0.03$ & $-22 \pm 1$ \\
\hline 0013+1942 & $32.58 \pm 3.49$ & $1.41 \pm 2.12$ & $19.85 \pm 0.07$ & $1.48 \pm 0.13$ & $0.00 \pm 0.04$ & $0.24 \pm 0.02$ & $52 \pm 6$ \\
\hline $0014+1829$ & $24.86 \pm 1.81$ & $14.96 \pm 3.84$ & $18.85 \pm 0.26$ & $1.02 \pm 0.08$ & $3.06 \pm 1.42$ & $0.30 \pm 0.06$ & $54 \pm 6$ \\
\hline $0014+1748$ & $20.18 \pm 0.43$ & $1.03 \pm 0.36$ & $22.26 \pm 0.11$ & $22.06 \pm 2.34$ & $0.05 \pm 0.01$ & $0.72 \pm 0.01$ & $46 \pm 1$ \\
\hline $0015+2212$ & $21.44 \pm 0.20$ & $1.71 \pm 0.38$ & $21.56 \pm 1.13$ & $2.30 \pm 0.63$ & $2.23 \pm \ldots$ & $0.09 \pm 0.05$ & $12 \pm 14$ \\
\hline $0017+1942$ & $23.00 \pm 0.89$ & $0.62 \pm 1.60$ & $20.18 \pm 0.04$ & $4.53 \pm 0.56$ & $0.00 \pm 0.01$ & $0.61 \pm 0.01$ & $1 \pm 2$ \\
\hline $0017+2148$ & $27.83 \pm 0.50$ & $0.81 \pm 0.46$ & $19.05 \pm 0.05$ & $1.23 \pm 0.06$ & $0.00 \pm 0.00$ & $0.22 \pm 0.04$ & $40 \pm 4$ \\
\hline $0018+2216$ & $22.18 \pm 0.37$ & $1.35 \pm 0.93$ & $19.86 \pm 0.22$ & $1.66 \pm 0.09$ & $0.28 \pm 0.43$ & $0.21 \pm 0.04$ & $-72 \pm 3$ \\
\hline $0018+2218$ & $26.59 \pm 0.16$ & $14.85 \pm 6.70$ & $21.73 \pm 0.23$ & $8.84 \pm 1.62$ & $0.12 \pm 0.14$ & $0.48 \pm 0.06$ & $-66 \pm 1$ \\
\hline $0019+2201$ & $22.76 \pm 0.35$ & $1.74 \pm 1.55$ & $20.51 \pm 0.34$ & $2.38 \pm 0.35$ & $0.24 \pm 0.62$ & $0.36 \pm 0.01$ & $69 \pm 3$ \\
\hline $0022+2049$ & $23.44 \pm 0.93$ & $1.26 \pm 1.69$ & $19.75 \pm 0.14$ & $3.56 \pm 0.37$ & $0.02 \pm 0.04$ & $0.51 \pm 0.02$ & $-63 \pm 1$ \\
\hline $0023+1908$ & - & - & - & - & - & $0.19 \pm 0.06$ & $40 \pm 3$ \\
\hline $0034+2119$ & $19.34 \pm 0.50$ & $0.25 \pm 0.15$ & $20.34 \pm 0.11$ & $3.63 \pm 0.29$ & $0.04 \pm 0.01$ & $0.44 \pm 0.06$ & $-85 \pm 2$ \\
\hline $0037+2226$ & $23.33 \pm 0.57$ & $3.46 \pm 2.78$ & $20.57 \pm 0.11$ & $8.18 \pm 0.57$ & $0.05 \pm 0.07$ & $0.07 \pm 0.03$ & $-22 \pm 11$ \\
\hline $0038+2259$ & $25.35 \pm 0.20$ & $5.76 \pm 2.06$ & $20.87 \pm 0.13$ & $5.33 \pm 0.92$ & $0.07 \pm 0.04$ & $0.60 \pm 0.03$ & $82 \pm 2$ \\
\hline $0039+0054$ & - & - & - & - & - & $0.31 \pm 0.07$ & $23 \pm 5$ \\
\hline $0040+0257$ & - & - & - & - & - & $0.35 \pm 0.04$ & $-26 \pm 4$ \\
\hline $0040+2312$ & $24.35 \pm 0.97$ & $0.43 \pm 0.53$ & $21.01 \pm 0.04$ & $7.77 \pm 0.63$ & $0.00 \pm 0.00$ & $0.66 \pm 0.03$ & $50 \pm 2$ \\
\hline $0040+0220$ & $23.59 \pm 3.20$ & $1.29 \pm 1.28$ & $19.29 \pm 0.13$ & $1.20 \pm 0.07$ & $0.08 \pm 0.07$ & $0.11 \pm 0.05$ & $-37 \pm 15$ \\
\hline 0040-0023 & $24.27 \pm 0.19$ & $7.35 \pm 1.75$ & $20.13 \pm 0.05$ & $8.32 \pm 0.64$ & $0.06 \pm 0.03$ & $0.24 \pm 0.00$ & $6 \pm 0$ \\
\hline $0041+0134$ & $26.02 \pm 0.20$ & $22.12 \pm 9.09$ & $22.31 \pm 0.27$ & $20.64 \pm 4.32$ & $0.14 \pm 0.18$ & $0.23 \pm 0.00$ & $70 \pm 0$ \\
\hline $0043+0245$ & $22.19 \pm 1.38$ & $0.48 \pm 0.31$ & $19.50 \pm 0.20$ & $1.32 \pm 0.19$ & $0.04 \pm 0.04$ & $0.20 \pm 0.09$ & $73 \pm 16$ \\
\hline 0043-0159 & $19.17 \pm 0.23$ & $1.23 \pm 0.21$ & $21.31 \pm 0.11$ & $20.71 \pm 1.26$ & $0.09 \pm 0.02$ & $0.34 \pm 0.05$ & $-30 \pm 6$ \\
\hline $0044+2246$ & $23.88 \pm 0.20$ & $3.35 \pm 1.02$ & $21.74 \pm 0.13$ & $9.96 \pm 2.70$ & $0.06 \pm 0.04$ & $0.68 \pm 0.01$ & $68 \pm 1$ \\
\hline $0045+2206$ & - & - & - & - & - & $0.27 \pm 0.03$ & $6 \pm 3$ \\
\hline $0047+2051$ & $20.60 \pm 0.45$ & $0.28 \pm 0.29$ & $20.05 \pm 0.10$ & $1.95 \pm 0.19$ & $0.04 \pm 0.01$ & $0.17 \pm 0.02$ & $-23 \pm 5$ \\
\hline 0047-0213 & $21.25 \pm 0.26$ & $2.63 \pm 0.53$ & $22.32 \pm 1.06$ & $8.82 \pm 2.33$ & $0.86 \pm \ldots$ & $0.40 \pm 0.11$ & $25 \pm 4$ \\
\hline $0047+2413$ & $22.26 \pm 0.32$ & $2.15 \pm 1.16$ & $21.34 \pm 0.30$ & $6.20 \pm 0.43$ & $0.19 \pm 0.22$ & $0.51 \pm 0.04$ & $40 \pm 4$ \\
\hline $0047+2414$ & $21.99 \pm 0.90$ & $0.57 \pm 1.52$ & $19.96 \pm 0.08$ & $5.42 \pm 0.46$ & $0.01 \pm 0.01$ & $0.27 \pm 0.02$ & $-74 \pm 4$ \\
\hline 0049-0006 & - & - & - & - & - & $0.33 \pm 0.05$ & $-76 \pm 5$ \\
\hline $0049+0017$ & $24.59 \pm 0.17$ & $9.66 \pm 1.33$ & $21.48 \pm 1.05$ & $1.62 \pm 2.80$ & $7.31 \pm \ldots$ & $0.40 \pm 0.05$ & $-19 \pm 2$ \\
\hline 0049-0045 & - & - & - & - & - & - & - \\
\hline $0050+0005$ & $23.12 \pm 0.27$ & $4.66 \pm 1.72$ & $20.61 \pm 0.66$ & $2.73 \pm 0.97$ & $1.04 \pm 2.21$ & $0.41 \pm 0.03$ & $73 \pm 3$ \\
\hline $0050+2114$ & - & - & - & - & - & - & - \\
\hline $0051+2430$ & $24.07 \pm 0.39$ & $8.79 \pm 4.14$ & $20.77 \pm 0.41$ & $4.87 \pm 1.20$ & $0.56 \pm 1.52$ & $0.43 \pm 0.09$ & $25 \pm 2$ \\
\hline 0054-0133 & $20.89 \pm 0.72$ & $0.31 \pm 1.21$ & $20.16 \pm 0.20$ & $3.47 \pm 0.75$ & $0.01 \pm 0.06$ & $0.36 \pm 0.03$ & $-71 \pm 3$ \\
\hline $0054+2337$ & $24.02 \pm 0.40$ & $5.39 \pm 3.36$ & $20.99 \pm 0.33$ & $10.85 \pm 2.12$ & $0.05 \pm 0.08$ & $0.72 \pm 0.03$ & $-7 \pm 2$ \\
\hline $0056+0044$ & $23.33 \pm 0.62$ & $1.41 \pm 1.11$ & $21.88 \pm 0.09$ & $5.80 \pm 0.91$ & $0.06 \pm 0.07$ & $0.54 \pm 0.02$ & $-30 \pm 2$ \\
\hline $0056+0043$ & - & - & - & - & - & $0.44 \pm 0.03$ & $82 \pm 3$ \\
\hline $0119+2156$ & $22.63 \pm 0.37$ & $0.82 \pm 1.15$ & $20.86 \pm 0.15$ & $3.63 \pm 0.59$ & $0.04 \pm 0.05$ & $0.68 \pm 0.03$ & $42 \pm 2$ \\
\hline $0121+2137$ & - & - & - & - & - & $0.29 \pm 0.07$ & $65 \pm 8$ \\
\hline $0129+2109$ & $22.51 \pm 0.66$ & $1.23 \pm 1.15$ & $20.95 \pm 0.17$ & $6.48 \pm 1.30$ & $0.03 \pm 0.03$ & $0.18 \pm 0.04$ & $-87 \pm 4$ \\
\hline $0134+2257$ & $20.58 \pm 0.34$ & $0.53 \pm 0.34$ & $21.09 \pm 0.16$ & $4.20 \pm 1.27$ & $0.09 \pm 0.06$ & $0.11 \pm 0.04$ & $23 \pm 0$ \\
\hline $0135+2242$ & $22.76 \pm 0.28$ & $3.72 \pm 0.85$ & $34.82 \pm 0.49$ & $5.41 \pm 0.19$ & $\ldots \pm \ldots$ & $0.22 \pm 0.09$ & $73 \pm 8$ \\
\hline
\end{tabular}


Table 2. continued

\begin{tabular}{|c|c|c|c|c|c|c|c|}
\hline $\begin{array}{l}\text { UCM name } \\
(1)\end{array}$ & $\begin{array}{l}\mu_{\mathrm{e}} \\
(2)\end{array}$ & $\begin{array}{l}r_{\mathrm{e}} \\
(3)\end{array}$ & $\begin{array}{l}\mu_{0} \\
(4)\end{array}$ & $\begin{array}{l}d_{\mathrm{L}} \\
(5)\end{array}$ & $\begin{array}{c}B / D \\
(6) \\
\end{array}$ & $\begin{array}{c}\epsilon \\
(7) \\
\end{array}$ & $\begin{array}{r}\mathrm{PA} \\
(8) \\
\end{array}$ \\
\hline $0138+2216$ & - & - & - & - & - & $0.55 \pm 0.02$ & $-53 \pm 1$ \\
\hline $0141+2220$ & $23.28 \pm 0.34$ & $2.61 \pm 1.55$ & $19.90 \pm 0.14$ & $3.21 \pm 0.19$ & $0.11 \pm 0.11$ & $0.64 \pm 0.03$ & $39 \pm 2$ \\
\hline $0142+2137$ & $21.41 \pm 0.60$ & $0.86 \pm 0.90$ & $21.67 \pm 0.16$ & $14.22 \pm 1.87$ & $0.02 \pm 0.02$ & $0.69 \pm 0.05$ & $36 \pm 4$ \\
\hline $0144+2519$ & - & - & - & - & - & $0.33 \pm 0.00$ & $47 \pm 0$ \\
\hline $0147+2309$ & $25.21 \pm 0.04$ & $16.29 \pm 0.00$ & $21.09 \pm 0.01$ & $2.61 \pm 0.02$ & $3.16 \pm 0.12$ & $0.54 \pm 0.03$ & $-3 \pm 3$ \\
\hline $0148+2124$ & $23.51 \pm 0.16$ & $3.38 \pm 0.45$ & $21.10 \pm 0.48$ & $1.82 \pm 0.70$ & $1.35 \pm 1.05$ & $0.20 \pm 0.11$ & $3 \pm 17$ \\
\hline $0150+2032$ & - & - & - & - & - & $0.52 \pm 0.00$ & $70 \pm 0$ \\
\hline $0156+2410$ & - & - & - & - & - & $0.50 \pm 0.03$ & $73 \pm 2$ \\
\hline $0157+2413$ & $25.00 \pm 0.48$ & $3.86 \pm 2.08$ & $20.82 \pm 0.06$ & $12.62 \pm 0.97$ & $0.01 \pm 0.00$ & $0.77 \pm 0.01$ & $-5 \pm 1$ \\
\hline $0157+2102$ & - & - & - & - & - & $0.58 \pm 0.04$ & $83 \pm 3$ \\
\hline $0159+2354$ & - & - & - & - & - & $0.45 \pm 0.06$ & $-37 \pm 5$ \\
\hline $0159+2326$ & $22.18 \pm 0.47$ & $0.49 \pm 0.58$ & $20.04 \pm 0.08$ & $3.26 \pm 0.23$ & $0.01 \pm 0.01$ & $0.29 \pm 0.02$ & $32 \pm 3$ \\
\hline $1246+2727$ & $22.38 \pm 0.38$ & $0.47 \pm 0.51$ & $20.62 \pm 0.11$ & $4.83 \pm 0.66$ & $0.01 \pm 0.00$ & $0.43 \pm 0.02$ & $-5 \pm 2$ \\
\hline $1247+2701$ & $23.43 \pm 2.56$ & $0.44 \pm 0.62$ & $20.46 \pm 0.16$ & $3.88 \pm 0.55$ & $0.00 \pm 0.01$ & $0.65 \pm 0.01$ & $51 \pm 1$ \\
\hline $1248+2912$ & $20.89 \pm 0.49$ & $0.61 \pm 0.85$ & $20.39 \pm 0.12$ & $4.75 \pm 0.78$ & $0.04 \pm 0.04$ & $0.32 \pm 0.02$ & $-71 \pm 3$ \\
\hline $1253+2756$ & $26.01 \pm 0.59$ & $16.82 \pm 2.27$ & $19.30 \pm 0.21$ & $1.98 \pm 0.14$ & $0.54 \pm 0.34$ & $0.31 \pm 0.03$ & $-11 \pm 3$ \\
\hline $1254+2741$ & - & - & - & - & - & $0.58 \pm 0.02$ & $-65 \pm 2$ \\
\hline $1254+2802$ & $25.26 \pm 1.88$ & $0.51 \pm 0.66$ & $20.85 \pm 0.03$ & $3.47 \pm 0.25$ & $0.00 \pm 0.00$ & $0.50 \pm 0.02$ & $75 \pm 2$ \\
\hline $1255+2819$ & - & - & - & - & - & $0.13 \pm 0.04$ & $50 \pm 10$ \\
\hline $1255+3125$ & - & - & - & - & - & $0.59 \pm 0.03$ & $1 \pm 2$ \\
\hline $1255+2734$ & - & - & - & - & - & $0.49 \pm 0.06$ & $-35 \pm 2$ \\
\hline $1256+2717$ & $23.94 \pm 0.38$ & $3.82 \pm 0.53$ & $20.70 \pm 0.57$ & $1.07 \pm 0.27$ & $2.33 \pm 5.18$ & $0.30 \pm 0.03$ & $54 \pm 4$ \\
\hline $1256+2732$ & $21.43 \pm 0.50$ & $1.73 \pm 1.15$ & $21.17 \pm 0.72$ & $4.12 \pm 1.46$ & $0.50 \pm 0.45$ & $0.34 \pm 0.06$ & $82 \pm 5$ \\
\hline $1256+2701$ & $22.59 \pm 0.68$ & $0.46 \pm 0.78$ & $21.71 \pm 0.09$ & $9.29 \pm 2.25$ & $0.00 \pm 0.00$ & $0.80 \pm 0.01$ & $-64 \pm 1$ \\
\hline $1256+2910$ & $22.01 \pm 0.38$ & $1.71 \pm 1.96$ & $21.47 \pm 0.75$ & $4.41 \pm 0.74$ & $0.33 \pm 2.58$ & $0.15 \pm 0.02$ & $21 \pm 7$ \\
\hline $1256+2823$ & - & - & - & - & - & $0.19 \pm 0.01$ & $31 \pm 5$ \\
\hline $1256+2754$ & $22.50 \pm 0.37$ & $6.89 \pm 2.20$ & $25.28 \pm 4.58$ & $6.36 \pm 2.20$ & $54.79 \pm \ldots$ & $0.15 \pm 0.06$ & $-80 \pm 10$ \\
\hline $1256+2722$ & $23.23 \pm 0.12$ & $0.76 \pm 0.16$ & $20.46 \pm 0.09$ & $3.35 \pm 0.39$ & $0.01 \pm 0.00$ & $0.64 \pm 0.01$ & $-48 \pm 1$ \\
\hline $1257+2808$ & $25.76 \pm 0.48$ & $13.13 \pm 4.87$ & $19.57 \pm 0.10$ & $1.96 \pm 0.13$ & $0.54 \pm 0.42$ & $0.29 \pm 0.02$ & $-6 \pm 2$ \\
\hline $1258+2754$ & $21.50 \pm 0.76$ & $1.12 \pm 2.79$ & $20.71 \pm 0.29$ & $4.53 \pm 0.61$ & $0.11 \pm 0.45$ & $0.37 \pm 0.07$ & $58 \pm 4$ \\
\hline $1259+2934$ & - & - & - & - & - & - & - \\
\hline $1259+3011$ & $20.29 \pm 0.30$ & $1.14 \pm 0.32$ & $21.22 \pm 0.47$ & $3.72 \pm 0.80$ & $0.80 \pm 1.02$ & $0.38 \pm 0.02$ & $30 \pm 2$ \\
\hline $1259+2755$ & $23.72 \pm 0.27$ & $7.82 \pm 3.83$ & $19.70 \pm 0.15$ & $3.37 \pm 0.22$ & $0.48 \pm 0.71$ & $0.45 \pm 0.05$ & $87 \pm 5$ \\
\hline $1300+2907$ & $22.96 \pm 0.31$ & $3.18 \pm 0.85$ & $20.92 \pm 0.37$ & $2.31 \pm 0.46$ & $1.16 \pm 0.66$ & $0.51 \pm 0.03$ & $54 \pm 2$ \\
\hline $1301+2904$ & $25.11 \pm 0.55$ & $8.96 \pm 5.73$ & $20.88 \pm 0.19$ & $4.49 \pm 0.39$ & $0.29 \pm 0.41$ & $0.13 \pm 0.02$ & $16 \pm 5$ \\
\hline $1302+2853$ & $21.98 \pm 0.95$ & $0.12 \pm 0.16$ & $19.66 \pm 0.07$ & $2.40 \pm 0.11$ & $0.00 \pm 0.01$ & $0.40 \pm 0.02$ & $85 \pm 5$ \\
\hline $1302+3032$ & $24.78 \pm 0.70$ & $9.06 \pm 5.99$ & $19.68 \pm 0.17$ & $1.55 \pm 0.18$ & $1.12 \pm 1.51$ & $0.34 \pm 0.02$ & $-55 \pm 2$ \\
\hline $1303+2908$ & $25.14 \pm 2.67$ & $0.31 \pm 1.41$ & $21.35 \pm 0.06$ & $4.79 \pm 0.54$ & $0.00 \pm 0.00$ & $0.57 \pm 0.04$ & $8 \pm 3$ \\
\hline $1304+2808$ & $28.17 \pm 0.10$ & $1.35 \pm 0.43$ & $20.52 \pm 0.01$ & $4.33 \pm 0.11$ & $0.00 \pm 0.00$ & $0.49 \pm 0.04$ & $-48 \pm 1$ \\
\hline $1304+2830$ & $24.87 \pm 2.86$ & $0.28 \pm 0.95$ & $19.79 \pm 0.15$ & $0.82 \pm 0.04$ & $0.00 \pm 0.17$ & $0.18 \pm 0.02$ & $86 \pm 4$ \\
\hline $1304+2907$ & $25.76 \pm 0.87$ & $18.14 \pm 1.25$ & $21.73 \pm 0.50$ & $8.62 \pm 0.60$ & $0.39 \pm 0.44$ & $0.20 \pm 0.00$ & $6 \pm 0$ \\
\hline $1304+2818$ & $23.74 \pm 0.53$ & $1.31 \pm 1.27$ & $20.65 \pm 0.08$ & $3.98 \pm 0.39$ & $0.02 \pm 0.02$ & $0.14 \pm 0.02$ & $54 \pm 2$ \\
\hline $1306+2938$ & $25.65 \pm 0.35$ & $19.09 \pm 4.05$ & $19.09 \pm 0.09$ & $2.46 \pm 0.26$ & $0.52 \pm 0.38$ & $0.18 \pm 0.07$ & $-18 \pm 11$ \\
\hline $1306+3111$ & $22.70 \pm 2.41$ & $0.16 \pm 0.38$ & $19.92 \pm 0.04$ & $2.26 \pm 0.12$ & $0.00 \pm 0.00$ & $0.19 \pm 0.03$ & $-63 \pm 5$ \\
\hline $1307+2910$ & $21.48 \pm 0.34$ & $1.50 \pm 0.73$ & $21.15 \pm 0.16$ & $15.72 \pm 3.58$ & $0.02 \pm 0.01$ & $0.57 \pm 0.02$ & $86 \pm 1$ \\
\hline $1308+2958$ & $21.22 \pm 0.35$ & $0.75 \pm 0.66$ & $21.58 \pm 0.15$ & $8.13 \pm 1.81$ & $0.04 \pm 0.02$ & $0.36 \pm 0.04$ & $0 \pm 4$ \\
\hline $1308+2950$ & - & - & - & - & - & $0.65 \pm 0.02$ & $-11 \pm 2$ \\
\hline
\end{tabular}


Table 2. continued

\begin{tabular}{|c|c|c|c|c|c|c|c|}
\hline $\begin{array}{l}\text { UCM name } \\
(1)\end{array}$ & $\begin{array}{l}\mu_{\mathrm{e}} \\
(2)\end{array}$ & $\begin{array}{l}r_{\mathrm{e}} \\
(3)\end{array}$ & $\begin{array}{l}\mu_{0} \\
(4)\end{array}$ & $\begin{array}{l}d_{\mathrm{L}} \\
(5)\end{array}$ & $\begin{array}{c}B / D \\
(6)\end{array}$ & $\begin{array}{c}\epsilon \\
(7)\end{array}$ & $\begin{array}{l}\mathrm{PA} \\
(8)\end{array}$ \\
\hline $1310+3027$ & $24.21 \pm 0.27$ & $5.13 \pm 1.65$ & $20.53 \pm 0.23$ & $3.11 \pm 0.43$ & $0.33 \pm 0.17$ & $0.56 \pm 0.02$ & $-31 \pm 2$ \\
\hline $1312+3040$ & $21.58 \pm 0.69$ & $1.50 \pm 1.40$ & $20.32 \pm 0.45$ & $3.73 \pm 0.66$ & $0.18 \pm 0.26$ & $0.35 \pm 0.09$ & $-84 \pm 3$ \\
\hline $1312+2954$ & - & - & - & - & & $0.62 \pm 0.06$ & $-82 \pm 1$ \\
\hline $1313+2938$ & $22.20 \pm 0.15$ & $2.06 \pm 1.62$ & $18.59 \pm 0.21$ & $0.95 \pm 0.05$ & $0.61 \pm 1.07$ & - & - \\
\hline $1314+2827$ & - & - & - & - & - & $0.01 \pm 0.04$ & - \\
\hline $1320+2727$ & $23.94 \pm 0.88$ & $4.41 \pm 0.59$ & $19.73 \pm 0.22$ & $1.10 \pm 0.12$ & $1.20 \pm 0.13$ & $0.32 \pm 0.05$ & $75 \pm 5$ \\
\hline $1324+2926$ & $24.91 \pm 0.45$ & $5.85 \pm 0.47$ & $19.62 \pm 0.21$ & $0.70 \pm 0.04$ & $1.93 \pm 1.23$ & $0.11 \pm 0.04$ & $54 \pm 21$ \\
\hline $1324+2651$ & $17.68 \pm 0.52$ & $0.53 \pm 0.23$ & $21.12 \pm 0.51$ & $5.36 \pm 1.25$ & $0.84 \pm 0.52$ & $0.33 \pm 0.01$ & $-24 \pm 1$ \\
\hline $1331+2900$ & - & - & - & - & - & $0.25 \pm 0.14$ & $-23 \pm 18$ \\
\hline $1428+2727$ & $25.90 \pm 3.09$ & $2.85 \pm 2.34$ & $19.22 \pm 0.11$ & $3.96 \pm 0.30$ & $0.00 \pm 0.02$ & $0.45 \pm 0.01$ & $83 \pm 1$ \\
\hline $1429+2645$ & $21.33 \pm 0.10$ & $0.32 \pm 0.12$ & $19.91 \pm 0.20$ & $1.14 \pm 0.12$ & $0.08 \pm 0.08$ & $0.11 \pm 0.04$ & $-41 \pm 18$ \\
\hline $1430+2947$ & $22.84 \pm 0.26$ & $4.45 \pm 0.80$ & $25.35 \pm 2.92$ & $11.47 \pm 4.24$ & $5.48 \pm \ldots$ & $0.23 \pm 0.04$ & $-87 \pm 4$ \\
\hline $1431+2854$ & $24.16 \pm 0.36$ & $8.65 \pm 3.92$ & $20.38 \pm 0.21$ & $3.36 \pm 0.32$ & $0.74 \pm 0.73$ & $0.21 \pm 0.04$ & $-68 \pm 5$ \\
\hline $1431+2702$ & $25.12 \pm 0.47$ & $10.05 \pm 2.03$ & $18.85 \pm 0.13$ & $0.77 \pm 0.05$ & $1.91 \pm 0.67$ & $0.10 \pm 0.06$ & $67 \pm 10$ \\
\hline $1431+2947$ & - & - & - & - & - & $0.39 \pm 0.03$ & $-50 \pm 5$ \\
\hline $1431+2814$ & $23.38 \pm 0.19$ & $2.30 \pm 1.57$ & $20.41 \pm 0.17$ & $2.93 \pm 0.23$ & $0.14 \pm 0.31$ & $0.65 \pm 0.01$ & $-25 \pm 1$ \\
\hline $1432+2645$ & $20.84 \pm 0.88$ & $1.23 \pm 1.09$ & $22.00 \pm 0.83$ & $10.04 \pm 3.64$ & $0.16 \pm 0.20$ & $0.42 \pm 0.01$ & $-78 \pm 6$ \\
\hline $1440+2521 \mathrm{~S}$ & - & - & - & - & - & $0.41 \pm 0.03$ & $24 \pm 2$ \\
\hline $1440+2511$ & $21.70 \pm 0.93$ & $1.04 \pm 0.83$ & $22.55 \pm 0.38$ & $8.15 \pm 3.20$ & $0.13 \pm 0.07$ & $0.31 \pm 0.09$ & $-24 \pm 3$ \\
\hline $1440+2521 \mathrm{~N}$ & $23.52 \pm 0.29$ & $3.00 \pm 1.38$ & $21.42 \pm 0.22$ & $4.53 \pm 0.52$ & $0.23 \pm 0.15$ & $0.41 \pm 0.05$ & $62 \pm 9$ \\
\hline $1442+2845$ & $25.64 \pm 0.29$ & $21.69 \pm 7.73$ & $20.34 \pm 0.13$ & $3.25 \pm 0.16$ & $1.22 \pm 1.05$ & $0.06 \pm 0.01$ & $-33 \pm 6$ \\
\hline $1443+2714$ & $22.14 \pm 0.13$ & $3.29 \pm 0.52$ & $21.24 \pm 0.35$ & $4.24 \pm 0.78$ & $0.95 \pm 0.37$ & $0.12 \pm 0.04$ & $72 \pm 24$ \\
\hline $1443+2844$ & $23.72 \pm 2.49$ & $0.13 \pm 1.10$ & $20.36 \pm 0.02$ & $3.98 \pm 0.38$ & $0.00 \pm 0.00$ & $0.49 \pm 0.01$ & $-58 \pm 1$ \\
\hline $1443+2548$ & $22.94 \pm 0.24$ & $1.19 \pm 0.64$ & $20.29 \pm 0.09$ & $3.88 \pm 0.37$ & $0.03 \pm 0.02$ & $0.21 \pm 0.03$ & $-82 \pm 8$ \\
\hline $1444+2923$ & $21.90 \pm 0.58$ & $1.84 \pm 1.15$ & $23.11 \pm 0.83$ & $7.06 \pm 2.57$ & $0.75 \pm 1.08$ & $0.12 \pm 0.06$ & $-13 \pm 11$ \\
\hline $1452+2754$ & $22.55 \pm 0.27$ & $2.49 \pm 0.74$ & $21.08 \pm 0.23$ & $3.50 \pm 0.48$ & $0.47 \pm 0.24$ & $0.51 \pm 0.01$ & $-44 \pm 1$ \\
\hline $1506+1922$ & $20.29 \pm 0.27$ & $0.71 \pm 0.25$ & $21.21 \pm 0.12$ & $5.22 \pm 0.33$ & $0.16 \pm 0.03$ & $0.43 \pm 0.01$ & $68 \pm 1$ \\
\hline $1513+2012$ & $27.47 \pm 0.44$ & $1.22 \pm 0.99$ & $19.09 \pm 0.03$ & $2.64 \pm 0.09$ & $0.00 \pm 0.00$ & $0.50 \pm 0.02$ & $55 \pm 2$ \\
\hline $1537+2506 \mathrm{~N}$ & $19.39 \pm 0.37$ & $0.74 \pm 0.19$ & $20.95 \pm 0.18$ & $5.91 \pm 0.57$ & $0.24 \pm 0.08$ & $0.35 \pm 0.05$ & $-69 \pm 5$ \\
\hline $1537+2506 \mathrm{~S}$ & $27.68 \pm 0.41$ & $74.55 \pm 9.86$ & $19.49 \pm 0.11$ & $2.06 \pm 0.30$ & $2.50 \pm 1.96$ & $0.44 \pm 0.04$ & $64 \pm 9$ \\
\hline $1557+1423$ & $24.84 \pm 3.84$ & $1.60 \pm 1.01$ & $20.06 \pm 0.12$ & $2.13 \pm 0.13$ & $0.02 \pm 0.05$ & $0.21 \pm 0.02$ & $35 \pm 3$ \\
\hline $1612+1308$ & - & - & - & - & - & $0.03 \pm 0.05$ & - \\
\hline $1646+2725$ & - & - & - & - & - & $0.68 \pm 0.02$ & $-70 \pm 1$ \\
\hline $1647+2950$ & $20.98 \pm 0.44$ & $0.89 \pm 0.74$ & $20.41 \pm 0.23$ & $3.85 \pm 0.52$ & $0.11 \pm 0.11$ & $0.20 \pm 0.01$ & $-33 \pm 2$ \\
\hline $1647+2729$ & $22.32 \pm 0.18$ & $0.52 \pm 0.55$ & $20.10 \pm 0.10$ & $3.49 \pm 0.29$ & $0.01 \pm 0.01$ & $0.43 \pm 0.02$ & $89 \pm 2$ \\
\hline $1647+2727$ & - & - & - & - & - & $0.44 \pm 0.01$ & $-90 \pm 1$ \\
\hline $1648+2855$ & $25.35 \pm 0.46$ & $15.02 \pm 3.16$ & $19.52 \pm 0.34$ & $2.08 \pm 0.18$ & $0.88 \pm 1.07$ & $0.16 \pm 0.04$ & $3 \pm 4$ \\
\hline $1653+2644$ & $21.60 \pm 0.71$ & $2.65 \pm 2.11$ & $19.19 \pm 0.24$ & $2.94 \pm 0.20$ & $0.32 \pm 0.57$ & $0.20 \pm 0.01$ & $1 \pm 2$ \\
\hline $1654+2812$ & $26.69 \pm 3.87$ & $0.88 \pm 1.47$ & $21.79 \pm 0.06$ & $2.96 \pm 0.36$ & $0.00 \pm 0.01$ & $0.60 \pm 0.02$ & $-49 \pm 2$ \\
\hline $1655+2755$ & $24.43 \pm 0.16$ & $6.48 \pm 2.06$ & $21.95 \pm 0.13$ & $10.83 \pm 1.67$ & $0.13 \pm 0.07$ & $0.49 \pm 0.10$ & $38 \pm 10$ \\
\hline $1656+2744$ & - & - & - & - & - & $0.48 \pm 0.09$ & $27 \pm 7$ \\
\hline $1657+2901$ & - & - & - & - & - & $0.51 \pm 0.01$ & $82 \pm 1$ \\
\hline $1659+2928$ & $19.76 \pm 0.32$ & $0.93 \pm 0.30$ & $21.64 \pm 0.39$ & $5.47 \pm 1.45$ & $0.59 \pm 0.15$ & $0.31 \pm 0.04$ & $-71 \pm 2$ \\
\hline $1701+3131$ & $19.24 \pm 0.16$ & $0.89 \pm 0.24$ & $21.41 \pm 0.35$ & $6.85 \pm 2.53$ & $0.45 \pm 0.24$ & $0.32 \pm 0.10$ & $59 \pm 8$ \\
\hline $2238+2308$ & $23.89 \pm 0.14$ & $13.24 \pm 2.45$ & $21.30 \pm 0.69$ & $6.16 \pm 2.60$ & $1.53 \pm 1.78$ & $0.20 \pm 0.03$ & $-6 \pm 4$ \\
\hline $2239+1959$ & $21.06 \pm 0.21$ & $4.00 \pm 0.47$ & $21.47 \pm 0.47$ & $4.95 \pm 1.26$ & $3.44 \pm 0.71$ & $0.35 \pm 0.02$ & $38 \pm 3$ \\
\hline $2249+2149$ & $22.44 \pm 0.27$ & $3.41 \pm 0.63$ & $22.04 \pm 0.35$ & $7.42 \pm 2.37$ & $0.53 \pm 0.15$ & $0.54 \pm 0.03$ & $58 \pm 2$ \\
\hline $2250+2427$ & $17.65 \pm 0.14$ & $0.47 \pm 0.04$ & $21.40 \pm 0.09$ & $10.50 \pm 0.15$ & $0.23 \pm 0.03$ & $0.51 \pm 0.06$ & $-21 \pm 2$ \\
\hline
\end{tabular}


Table 2. continued

\begin{tabular}{|c|c|c|c|c|c|c|c|}
\hline $\begin{array}{l}\text { UCM name } \\
\text { (1) }\end{array}$ & $\begin{array}{l}\mu_{\mathrm{e}} \\
(2) \\
\end{array}$ & $\begin{array}{l}r_{\mathrm{e}} \\
(3)\end{array}$ & $\begin{array}{l}\mu_{0} \\
(4) \\
\end{array}$ & $\begin{array}{l}d_{\mathrm{L}} \\
(5)\end{array}$ & $\begin{array}{c}B / D \\
(6) \\
\end{array}$ & $\begin{array}{c}\epsilon \\
(7) \\
\end{array}$ & $\begin{array}{l}\mathrm{PA} \\
(8) \\
\end{array}$ \\
\hline $2251+2352$ & $21.31 \pm 3.85$ & $0.11 \pm 1.37$ & $18.71 \pm 0.05$ & $1.27 \pm 0.05$ & $0.00 \pm 0.01$ & $0.02 \pm 0.03$ & - \\
\hline $2253+2219$ & $22.48 \pm 1.09$ & $1.09 \pm 1.56$ & $19.11 \pm 0.12$ & $2.31 \pm 0.10$ & $0.04 \pm 0.08$ & $0.56 \pm 0.02$ & $32 \pm 1$ \\
\hline $2255+1930 \mathrm{~S}$ & $21.11 \pm 1.48$ & $0.43 \pm 0.53$ & $18.51 \pm 0.26$ & $1.37 \pm 0.13$ & $0.03 \pm 0.14$ & $0.30 \pm 0.05$ & $41 \pm 4$ \\
\hline $2255+1930 \mathrm{~N}$ & $24.35 \pm 0.20$ & $6.98 \pm 2.63$ & $19.87 \pm 0.15$ & $3.29 \pm 0.20$ & $0.26 \pm 0.28$ & $0.55 \pm 0.01$ & $-87 \pm 1$ \\
\hline $2255+1926$ & $26.24 \pm 0.20$ & $15.81 \pm 2.28$ & $21.36 \pm 0.19$ & $3.89 \pm 0.66$ & $0.67 \pm 0.18$ & $0.57 \pm 0.03$ & $-17 \pm 3$ \\
\hline $2255+1654$ & $26.26 \pm 0.23$ & $9.89 \pm 5.32$ & $21.45 \pm 0.17$ & $8.22 \pm 1.60$ & $0.06 \pm 0.08$ & $0.76 \pm 0.01$ & $73 \pm 1$ \\
\hline $2256+2001$ & $23.66 \pm 0.64$ & $1.49 \pm 1.42$ & $22.23 \pm 0.14$ & $10.81 \pm 2.03$ & $0.02 \pm 0.01$ & $0.20 \pm 0.02$ & $-5 \pm 2$ \\
\hline $2257+2438$ & $19.31 \pm 0.09$ & $1.10 \pm 0.06$ & $27.33 \pm 3.30$ & $2.80 \pm 0.71$ & $\ldots \pm \ldots$ & $0.20 \pm 0.03$ & $-55 \pm 5$ \\
\hline $2257+1606$ & $20.44 \pm 0.36$ & $1.07 \pm 0.43$ & $21.09 \pm 1.16$ & $2.35 \pm 1.25$ & $1.36 \pm \ldots$ & $0.06 \pm 0.07$ & - \\
\hline $2258+1920$ & - & - & - & - & - & $0.23 \pm 0.04$ & $72 \pm 5$ \\
\hline $2300+2015$ & - & - & - & - & - & $0.08 \pm 0.03$ & $-64 \pm 14$ \\
\hline $2302+2053 \mathrm{~W}$ & $23.47 \pm 0.38$ & $3.23 \pm 1.74$ & $22.40 \pm 1.12$ & $5.13 \pm 1.39$ & $0.53 \pm \ldots$ & $0.62 \pm 0.01$ & $66 \pm 1$ \\
\hline $2302+2053 \mathrm{E}$ & $23.33 \pm 0.17$ & $5.17 \pm 0.85$ & $21.76 \pm 0.29$ & $6.92 \pm 1.19$ & $0.47 \pm 0.23$ & $0.30 \pm 0.02$ & $32 \pm 9$ \\
\hline $2303+1856$ & - & - & - & - & - & $0.41 \pm 0.07$ & $-24 \pm 7$ \\
\hline $2303+1702$ & $24.79 \pm 0.57$ & $2.10 \pm 0.62$ & $21.24 \pm 0.19$ & $2.70 \pm 0.49$ & $0.08 \pm 0.02$ & $0.21 \pm 0.08$ & $48 \pm 3$ \\
\hline $2304+1640$ & - & - & - & - & - & $0.23 \pm 0.07$ & $-50 \pm 14$ \\
\hline $2304+1621$ & $20.30 \pm 0.03$ & $0.56 \pm 0.02$ & $21.21 \pm 0.04$ & $2.86 \pm 0.01$ & $0.32 \pm 0.02$ & $0.31 \pm 0.07$ & $-6 \pm 5$ \\
\hline $2307+1947$ & $20.50 \pm 0.65$ & $0.65 \pm 0.97$ & $21.07 \pm 0.82$ & $3.33 \pm 0.46$ & $0.23 \pm 2.01$ & $0.43 \pm 0.01$ & $-85 \pm 1$ \\
\hline $2310+1800$ & $21.45 \pm 0.26$ & $0.59 \pm 1.02$ & $20.39 \pm 0.23$ & $2.49 \pm 0.23$ & $0.08 \pm 0.24$ & $0.32 \pm 0.15$ & $14 \pm 11$ \\
\hline $2312+2204$ & $21.11 \pm 0.23$ & $0.61 \pm 0.78$ & $20.70 \pm 0.56$ & $2.15 \pm 0.79$ & $0.20 \pm 2.80$ & $0.37 \pm 0.04$ & $-24 \pm 4$ \\
\hline $2313+1841$ & - & - & - & - & - & $0.57 \pm 0.04$ & $-33 \pm 4$ \\
\hline $2313+2517$ & $20.93 \pm 0.19$ & $1.37 \pm 0.45$ & $20.07 \pm 0.15$ & $4.49 \pm 0.38$ & $0.15 \pm 0.08$ & $0.36 \pm 0.02$ & $7 \pm 1$ \\
\hline $2315+1923$ & $19.48 \pm 0.12$ & $0.23 \pm 0.12$ & $20.62 \pm 0.12$ & $2.15 \pm 0.02$ & $0.12 \pm 0.05$ & $0.50 \pm 0.06$ & $-4 \pm 5$ \\
\hline $2316+2457$ & $21.08 \pm 0.17$ & $3.53 \pm 0.31$ & $20.95 \pm 0.31$ & $5.79 \pm 0.22$ & $1.19 \pm 0.31$ & $0.02 \pm 0.02$ & - \\
\hline $2316+2459$ & - & - & - & - & - & $0.26 \pm 0.03$ & $28 \pm 4$ \\
\hline $2316+2028$ & - & - & - & - & - & $0.28 \pm 0.06$ & $32 \pm 12$ \\
\hline $2317+2356$ & $22.11 \pm 0.53$ & $5.17 \pm 3.13$ & $20.51 \pm 0.48$ & $9.03 \pm 2.56$ & $0.27 \pm 0.34$ & $0.22 \pm 0.03$ & $-73 \pm 5$ \\
\hline $2319+2234$ & $21.97 \pm 1.17$ & $0.47 \pm 0.61$ & $19.54 \pm 0.15$ & $1.86 \pm 0.25$ & $0.02 \pm 0.02$ & $0.41 \pm 0.04$ & $88 \pm 3$ \\
\hline $2319+2243$ & $22.38 \pm 0.41$ & $3.35 \pm 1.43$ & $20.89 \pm 0.52$ & $4.04 \pm 1.16$ & $0.63 \pm 0.47$ & $0.41 \pm 0.08$ & $-29 \pm 4$ \\
\hline $2320+2428$ & $21.60 \pm 0.49$ & $0.41 \pm 0.55$ & $20.11 \pm 0.08$ & $4.59 \pm 0.55$ & $0.01 \pm 0.01$ & $0.73 \pm 0.03$ & $8 \pm 1$ \\
\hline $2321+2149$ & $23.77 \pm 0.50$ & $1.40 \pm 1.32$ & $20.19 \pm 0.26$ & $2.30 \pm 0.38$ & $0.05 \pm 0.10$ & $0.12 \pm 0.02$ & $-51 \pm 7$ \\
\hline $2321+2506$ & $21.51 \pm 0.78$ & $0.25 \pm 0.89$ & $20.73 \pm 0.07$ & $5.82 \pm 0.42$ & $0.00 \pm 0.01$ & $0.50 \pm 0.02$ & $60 \pm 5$ \\
\hline $2322+2218$ & $22.59 \pm 0.87$ & $0.90 \pm 0.72$ & $21.01 \pm 0.19$ & $3.08 \pm 0.48$ & $0.07 \pm 0.04$ & $0.56 \pm 0.01$ & $-31 \pm 1$ \\
\hline $2324+2448$ & $22.57 \pm 0.49$ & $2.40 \pm 2.54$ & $20.07 \pm 0.10$ & $12.57 \pm 1.15$ & $0.01 \pm 0.02$ & $0.58 \pm 0.01$ & $-83 \pm 1$ \\
\hline $2325+2318$ & - & - & - & - & - & - & - \\
\hline $2325+2208$ & - & - & - & - & - & $0.18 \pm 0.03$ & $36 \pm 1$ \\
\hline $2326+2435$ & - & - & - & - & - & $0.74 \pm 0.02$ & $-73 \pm 1$ \\
\hline $2327+2515 \mathrm{~N}$ & $20.77 \pm 0.46$ & $0.97 \pm 0.92$ & $19.07 \pm 0.20$ & $2.45 \pm 0.24$ & $0.12 \pm 0.15$ & $0.43 \pm 0.08$ & $-1 \pm 5$ \\
\hline $2327+2515 \mathrm{~S}$ & $21.00 \pm 0.44$ & $1.83 \pm 1.03$ & $21.53 \pm 0.64$ & $5.94 \pm 2.68$ & $0.56 \pm 9.90$ & $0.20 \pm 0.05$ & $33 \pm 5$ \\
\hline $2329+2427$ & $21.31 \pm 0.22$ & $0.86 \pm 0.26$ & $21.29 \pm 0.14$ & $6.20 \pm 1.30$ & $0.07 \pm 0.03$ & $0.62 \pm 0.03$ & $-58 \pm 1$ \\
\hline $2329+2500$ & $19.83 \pm 0.23$ & $0.88 \pm 0.18$ & $23.17 \pm 0.52$ & $13.92 \pm 0.75$ & $0.31 \pm 0.56$ & $0.36 \pm 0.04$ & $-65 \pm 2$ \\
\hline $2329+2512$ & $24.86 \pm 2.67$ & $6.47 \pm 0.43$ & $19.90 \pm 0.22$ & $1.17 \pm 0.07$ & $1.14 \pm 0.64$ & $0.26 \pm 0.07$ & $70 \pm 6$ \\
\hline $2331+2214$ & $21.74 \pm 0.42$ & $0.76 \pm 0.41$ & $21.95 \pm 0.29$ & $3.36 \pm 0.73$ & $0.22 \pm 0.15$ & $0.43 \pm 0.04$ & $14 \pm 1$ \\
\hline $2333+2248$ & $21.52 \pm 0.79$ & $0.15 \pm 0.58$ & $21.54 \pm 0.08$ & $5.02 \pm 0.85$ & $0.00 \pm 0.00$ & $0.72 \pm 0.04$ & $-64 \pm 3$ \\
\hline $2333+2359$ & $24.09 \pm 0.24$ & $4.87 \pm 1.12$ & $23.27 \pm 1.72$ & $1.65 \pm 1.05$ & $14.77 \pm$ & $0.12 \pm 0.08$ & $-20 \pm 21$ \\
\hline $2348+2407$ & - & - & - & - & - & $0.28 \pm 0.05$ & $25 \pm 7$ \\
\hline $2351+2321$ & $21.51 \pm 0.32$ & $0.57 \pm 0.34$ & $21.60 \pm 0.26$ & $1.40 \pm 0.39$ & $0.65 \pm 4.44$ & $0.13 \pm 0.05$ & $61 \pm 11$ \\
\hline
\end{tabular}

(1) UCM name. (2) Effective surface brightness of the bulge in mag $\operatorname{arcsec}^{-2}$. (3) Scale of the bulge in arcsec. (4) Characteristic surface brightness of the disk in mag $\operatorname{arcsec}^{-2}$. (5) Exponential disk scale in arcsec. (6) Bulge-to-disk ratio. (7) Ellipticity of the galaxy calculated as an average of the ellipticities of the isophotes between 23 and $24 \mathrm{mag}^{-\operatorname{arcsec}^{-2}}$. (8) Position angle in degrees measured counterclockwise from the North axis and calculated as an average of the previously detailed isophotes. 
where $\mu_{\mathrm{L}}$ is the detection threshold (set to $24.5 \mathrm{mag} \operatorname{arcsec}^{-2}$, mean value in our images) and $\alpha$ a parameter $0<\alpha<1$, appropriately chosen (it was set to 0.3 , optimal value as described in Doi et al. 1993).

Mean surface brightnesses, radii (calculated as the semimajor axis of the isophote) and magnitudes inside the $24.5 \mathrm{mag} \mathrm{arcsec}^{-2}$ isophote, effective radii (see Paper I) and mean surface brightnesses inside the effective isophote have also been calculated.

All these parameters are listed in Table 3. It was not possible to obtain reliable parameters for two objects, since their images were of very bad quality.

\subsection{Asymmetry parameter}

An asymmetry parameter $A$ was computed for each galaxy according to the definition established by Abraham et al. (1996). Each image was first smoothed with a Gaussian kernel of $\sigma=1$ pixel. After smoothing, it was rotated $180^{\circ}$ around the center of the object (this center was determined as the average of the inner isophotes of the galaxy). Finally, the rotated image was subtracted from the original. The parameter $A$ was calculated as:

$A=\frac{\Sigma\left|\left(I_{0}-I_{180}\right)\right|}{2 \Sigma\left|I_{0}\right|}$

where the sum runs over all the pixels, $I_{0}$ is the intensity of the original smoothed image and $I_{180}$ the intensity of the rotated one. Since the absolute value of the flux of the self-subtracted image is used, the uncertainty in the sky value adds a positive $A$ signal. This effect was corrected calculating the parameter $A$ for a region of the sky of the same size of the galaxy aperture and then subtracting it from the one calculated for the galaxy. This coefficient is shown in Table 3.

\section{Result discussion}

\subsection{Bulge-disk parameters}

Bulge-disk decomposition has been performed for a total number of 147 objects ( $77 \%$ of the sample). The rest of the galaxy profiles were very distorted or the images did not present enough quality to attempt the fitting. None of the morphological types is segregated from this subsample.

Figure 1 shows the histogram of the bulge-to-disk luminosity ratio for the UCM Survey galaxies in the Johnson $B$ band; dotted lines in this picture and the next ones stand for the Gunn $r$ data (Vitores et al. 1996a). Median values (the upper corresponds to $B$ and the lower to $r$ ) and error bars referring to the first quartiles (black line for blue data and grey line for red results) are shown at the top. The mean $B / D$ value is 0.40 with a standard deviation of 0.65 . This ratio is common for a $\mathrm{Sb}-\mathrm{Sbc}$ galaxy, according to Kent (1985). Special care should be taken with the $B / D$ ratio when classifying galaxies, particularly when $B / D \geq 1.7$ (14 of our galaxies have a bulge-to-disk

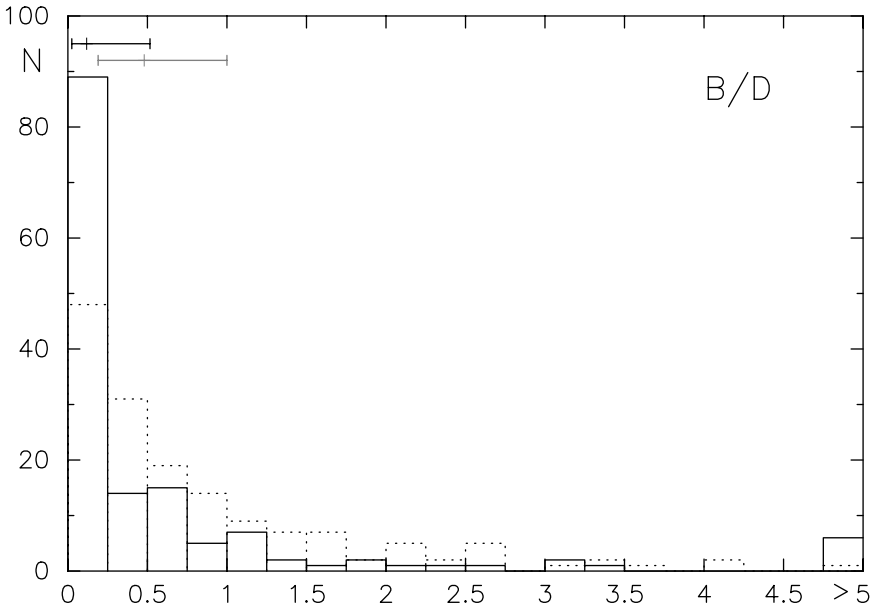

Fig. 1. Bulge-to-disk ratio histogram of the UCM Survey in the Johnson $B$ band. In this plot and hereafter, the median value and first quartiles of the data will be shown at the top. Black lines correspond to $B$ band results; dotted lines for the histograms and grey lines for the median and error bars will refer to the Gunn $r$ data from now on

ratio above this value); based on this statement, this criterium has only been taken into account in galaxy profiles easily separable into clear bulge and disk components, where the concept of $B / D$ ratio is meaningful (Simien \& de Vaucouleurs 1986; Schombert \& Bothun 1987).

Overall, bulge-to-disk ratios based on $B$ images are lower than those calculated with the Gunn $r$ data. The difference could be, in part, due to the distinct methods used to fit the surface brightness profiles, being the seeing convolution treatment the main difference. We have performed a test on the artificial galaxies introduced in Sect. 3.2 not taking into account the seeing-dominated zone of the profiles; the bulge-to-disk ratios calculated in this case are, in average, $\sim 10 \%$ larger than the values achieved using the seeing convolution. Therefore, the effect of seeing does not seem to cope with the whole difference between the $B$ and $r$ bulge-to-disk ratios; on the contrary, it appears to be a real characteristic of the objects.

In Figs. 2-5, we show the histograms for the bulge and disk parameters $\mu_{\mathrm{e}}^{\mathrm{c}}, r_{\mathrm{e}}, \mu_{0}^{\mathrm{c}}$ and $d_{\mathrm{L}}^{\mathrm{c}}$, respectively. Scales are in kpc and surface brightnesses in mag $\operatorname{arcsec}^{-2}$. Superindex c denotes correction for Galactic extinction and inclination (in the disk typical surface brightness).

The averaged $\mu_{\mathrm{e}}^{\mathrm{c}}$ value is $22.8 \pm 2.3 \mathrm{mag} \mathrm{arcsec}^{-2}$ $\left(22.7 \pm 2.3 \mathrm{mag} \operatorname{arcsec}^{-2}\right.$ if we only take into account the galaxies with $B / D<1.7$, the low $B / D$ subsample hereafter), typical for a late-type spiral (Kent 1985; Simien 1989). The typical scale of the bulge is in average $2.7 \mathrm{kpc}$ (2.2 kpc for the low- $B / D$ subsample), with a great dispersion $(\sigma=4.8)$, but also common for a Sb-Sc galaxy. These values are very similar to the ones measured in the Gunn $r$ images, although there seems to be a lack of small bulges in the red data.

The histogram of the characteristic surface brightness of the disk (Fig. 4) is dominated by galaxies with 
Table 3. Luminosity parameters for the UCM galaxies

\begin{tabular}{|c|c|c|c|c|c|c|c|c|}
\hline $\begin{array}{l}\text { UCM name } \\
\text { (1) }\end{array}$ & $\begin{array}{l}c_{31} \\
(2)\end{array}$ & $\begin{array}{l}c_{42} \\
(3)\end{array}$ & $\begin{array}{l}c_{\text {in }} \\
(4)\end{array}$ & $\begin{array}{c}b_{24.5} \\
(5) \\
\end{array}$ & $\begin{array}{c}r_{24.5} \\
(6)\end{array}$ & $\begin{array}{c}<\mu_{24.5}> \\
(7)\end{array}$ & $\begin{array}{c}<\mu_{\mathrm{e}}> \\
(8)\end{array}$ & $\begin{array}{c}A \\
(9)\end{array}$ \\
\hline $0000+2140$ & 6.59 & 4.98 & 0.54 & 14.67 & 19.2 & 22.86 & 20.87 & 0.19 \\
\hline $0003+2200$ & 2.25 & 2.31 & 0.23 & 17.18 & 5.8 & 22.49 & 21.53 & 0.03 \\
\hline $0003+2215$ & 2.62 & 2.60 & 0.21 & 15.93 & 12.5 & 22.66 & 21.58 & 0.09 \\
\hline $0003+1955$ & 2.98 & 3.28 & 0.90 & 14.12 & 9.2 & 20.63 & 16.04 & 0.11 \\
\hline $0005+1802$ & 3.28 & 3.24 & 0.47 & 16.47 & 8.8 & 22.56 & 21.27 & 0.04 \\
\hline $0006+2332$ & 3.04 & 2.99 & 0.33 & 15.04 & 11.7 & 22.56 & 21.39 & 0.31 \\
\hline $0013+1942$ & 3.10 & 3.10 & 0.32 & 17.17 & 5.6 & 22.69 & 21.57 & 0.21 \\
\hline $0014+1829$ & 4.24 & 3.92 & 0.53 & 16.25 & 5.4 & 22.40 & 20.59 & 0.37 \\
\hline $0014+1748$ & 2.74 & 2.91 & 0.30 & 15.05 & 20.8 & 23.18 & 22.62 & 0.09 \\
\hline $0015+2212$ & 4.21 & 3.85 & 0.59 & 16.81 & 5.0 & 22.51 & 20.65 & 0.00 \\
\hline $0017+1942$ & 2.64 & 2.69 & 0.27 & 15.88 & 12.6 & 22.52 & 21.35 & 0.23 \\
\hline $0017+2148$ & 5.02 & 4.45 & 0.50 & 17.01 & 4.1 & 22.46 & 20.84 & 0.01 \\
\hline $0018+2216$ & 3.25 & 3.15 & 0.47 & 16.87 & 3.5 & 22.27 & 20.56 & 0.06 \\
\hline $0018+2218$ & 2.82 & 2.93 & 0.40 & 16.13 & 14.9 & 23.18 & 22.66 & 0.18 \\
\hline $0019+2201$ & 4.62 & 4.13 & 0.45 & 16.77 & 6.1 & 22.86 & 21.86 & 0.02 \\
\hline $0022+2049$ & 2.97 & 2.99 & 0.49 & 15.74 & 8.1 & 22.23 & 20.68 & 0.13 \\
\hline $0023+1908$ & 2.34 & 2.36 & 0.40 & 16.88 & 4.5 & 21.85 & 20.50 & 0.04 \\
\hline $0034+2119$ & 3.58 & 3.43 & 0.47 & 15.98 & 16.7 & 22.90 & 21.51 & 0.09 \\
\hline $0037+2226$ & 2.54 & 2.53 & 0.12 & 14.68 & 10.7 & 22.29 & 21.20 & 0.10 \\
\hline $0038+2259$ & 2.88 & 2.88 & 0.32 & 16.28 & 21.5 & 22.89 & 21.77 & 0.08 \\
\hline $0039+0054$ & 2.59 & 2.76 & 0.23 & 15.23 & 12.2 & 22.92 & 22.38 & 0.10 \\
\hline $0040+0257$ & 2.78 & 2.92 & 0.35 & 17.00 & 7.1 & 21.93 & 20.44 & 0.15 \\
\hline $0040+2312$ & 3.11 & 3.09 & 0.33 & 15.86 & 17.8 & 22.96 & 22.00 & 0.11 \\
\hline $0040+0220$ & 2.76 & 2.90 & 0.32 & 17.19 & 2.7 & 22.05 & 20.48 & 0.06 \\
\hline $0040-0023$ & 3.11 & 3.15 & 0.41 & 13.80 & 15.1 & 22.62 & 21.31 & 0.09 \\
\hline $0041+0134$ & 2.49 & 2.51 & 0.25 & 14.49 & 18.5 & 23.40 & 22.88 & 0.09 \\
\hline $0043+0245$ & 2.83 & 3.08 & 0.36 & 17.33 & 3.0 & 22.23 & 20.74 & 0.05 \\
\hline $0043-0159$ & 2.11 & 2.17 & 0.27 & 13.07 & 21.3 & 22.22 & 21.21 & 0.20 \\
\hline $0044+2246$ & 2.74 & 2.84 & 0.34 & 16.20 & 16.3 & 23.01 & 22.21 & 0.12 \\
\hline $0045+2206$ & 2.52 & 2.57 & 0.57 & 15.07 & 7.7 & 21.52 & 19.61 & 0.10 \\
\hline $0047+2051$ & 2.40 & 2.37 & 0.32 & 16.90 & 10.3 & 22.15 & 20.93 & 0.08 \\
\hline $0047-0213$ & 6.24 & 4.79 & 0.61 & 15.66 & 6.9 & 22.50 & 20.43 & 0.03 \\
\hline $0047+2413$ & 3.17 & 3.24 & 0.37 & 15.93 & 18.2 & 22.81 & 21.63 & 0.13 \\
\hline $0047+2414$ & 2.63 & 2.61 & 0.34 & 15.27 & 13.9 & 21.99 & 20.72 & 0.13 \\
\hline 0049-0006 & 3.39 & 3.77 & 0.35 & 18.74 & 3.6 & 22.28 & 21.01 & 0.01 \\
\hline $0049+0017$ & 4.24 & 3.87 & 0.51 & 17.32 & 3.9 & 22.83 & 21.79 & 0.05 \\
\hline 0049-0045 & 3.38 & 3.31 & 0.48 & 15.33 & 2.2 & 22.36 & 20.74 & 0.29 \\
\hline $0050+0005$ & 3.44 & 3.35 & 0.51 & 16.42 & 10.4 & 22.32 & 20.75 & 0.11 \\
\hline $0050+2114$ & - & - & - & - & - & - & - & - \\
\hline $0051+2430$ & 3.42 & 3.29 & 0.52 & 15.32 & 12.4 & 22.78 & 21.49 & 0.05 \\
\hline $0054-0133$ & 3.35 & 3.50 & 0.34 & 16.04 & 18.3 & 22.43 & 21.49 & 0.12 \\
\hline $0054+2337$ & 4.05 & 3.74 & 0.45 & 15.40 & 17.5 & 23.17 & 21.88 & 0.16 \\
\hline $0056+0044$ & 3.43 & 3.45 & 0.32 & 17.07 & 8.0 & 23.43 & 22.98 & 0.12 \\
\hline $0056+0043$ & 2.59 & 2.66 & 0.40 & 16.61 & 4.6 & 22.05 & 20.41 & 0.06 \\
\hline $0119+2156$ & 3.06 & 3.02 & 0.38 & 16.75 & 27.4 & 22.98 & 22.22 & 0.08 \\
\hline $0121+2137$ & 2.80 & 2.69 & 0.26 & 15.93 & 19.0 & 23.26 & 22.43 & 0.11 \\
\hline $0129+2109$ & 2.53 & 2.57 & 0.17 & 15.19 & 19.8 & 22.79 & 21.75 & 0.09 \\
\hline $0134+2257$ & 3.72 & 3.54 & 0.35 & 16.16 & 14.6 & 23.17 & 22.21 & 0.02 \\
\hline
\end{tabular}


Table 3. continued

\begin{tabular}{|c|c|c|c|c|c|c|c|c|}
\hline $\begin{array}{l}\text { UCM name } \\
\text { (1) }\end{array}$ & $\begin{array}{l}c_{31} \\
(2) \\
\end{array}$ & $\begin{array}{l}c_{42} \\
(3)\end{array}$ & $\begin{array}{l}c_{\text {in }} \\
(4)\end{array}$ & $\begin{array}{c}b_{24.5} \\
(5)\end{array}$ & $\begin{array}{c}r_{24.5} \\
(6) \\
\end{array}$ & $\begin{array}{c}<\mu_{24.5}> \\
(7)\end{array}$ & $\begin{array}{c}<\mu_{\mathrm{e}}> \\
(8)\end{array}$ & $\begin{array}{c}A \\
(9) \\
\end{array}$ \\
\hline $0135+2242$ & 5.56 & 4.59 & 0.56 & 17.15 & 7.7 & 22.54 & 21.21 & 0.02 \\
\hline $0138+2216$ & 2.77 & 2.88 & 0.15 & 17.78 & 10.1 & 22.49 & 21.30 & 0.12 \\
\hline $0141+2220$ & 3.19 & 2.83 & 0.46 & 16.32 & 7.1 & 22.33 & 20.63 & 0.06 \\
\hline $0142+2137$ & 3.03 & 3.10 & 0.34 & 15.61 & 34.3 & 23.30 & 22.35 & 0.07 \\
\hline $0144+2519$ & 2.36 & 2.33 & 0.16 & 15.77 & 22.5 & 23.08 & 22.35 & 0.11 \\
\hline $0147+2309$ & 3.32 & 3.28 & 0.50 & 16.86 & 7.0 & 22.72 & 21.36 & 0.01 \\
\hline $0148+2124$ & 3.95 & 3.79 & 0.31 & 17.21 & 3.2 & 22.50 & 21.34 & 0.04 \\
\hline $0150+2032$ & 2.55 & 2.57 & 0.24 & 16.91 & 12.6 & 23.11 & 22.55 & 0.15 \\
\hline $0156+2410$ & 3.06 & 3.01 & 0.38 & 15.28 & 7.5 & 22.26 & 20.77 & 0.07 \\
\hline $0157+2413$ & 2.61 & 2.62 & 0.35 & 15.13 & 20.7 & 22.84 & 21.73 & 0.11 \\
\hline $0157+2102$ & 2.59 & 2.73 & 0.35 & 14.92 & 6.0 & 21.78 & 20.09 & 0.11 \\
\hline $0159+2354$ & 3.48 & 3.33 & 0.42 & 17.34 & 3.2 & 22.42 & 21.05 & 0.01 \\
\hline $0159+2326$ & 2.94 & 2.93 & 0.35 & 15.97 & 7.3 & 22.59 & 21.27 & 0.04 \\
\hline $1246+2727$ & 2.53 & 2.57 & 0.30 & 15.90 & 9.3 & 22.64 & 21.59 & 0.11 \\
\hline $1247+2701$ & 2.68 & 2.64 & 0.33 & 16.74 & 7.8 & 22.42 & 21.26 & 0.13 \\
\hline $1248+2912$ & 2.91 & 3.03 & 0.21 & 15.14 & 11.1 & 22.46 & 21.53 & 0.10 \\
\hline $1253+2756$ & 3.00 & 2.99 & 0.50 & 15.94 & 5.0 & 21.98 & 20.36 & 0.20 \\
\hline $1254+2741$ & 3.64 & 3.52 & 0.37 & 17.05 & 6.0 & 23.02 & 22.20 & 0.03 \\
\hline $1254+2802$ & 2.47 & 2.44 & 0.24 & 16.93 & 8.1 & 22.71 & 21.65 & 0.02 \\
\hline $1255+2819$ & 3.74 & 3.53 & 0.38 & 15.92 & 13.5 & 23.21 & 22.54 & 0.09 \\
\hline $1255+3125$ & 4.74 & 4.29 & 0.62 & 16.36 & 11.0 & 22.47 & 20.65 & 0.13 \\
\hline $1255+2734$ & 2.72 & 2.83 & 0.24 & 16.90 & 6.9 & 22.62 & 21.63 & 0.09 \\
\hline $1256+2717$ & 5.02 & 4.37 & 0.52 & 18.01 & 5.0 & 22.87 & 21.64 & 0.02 \\
\hline $1256+2732$ & 3.74 & 3.44 & 0.49 & 16.10 & 8.3 & 22.63 & 21.13 & 0.08 \\
\hline $1256+2701$ & 2.51 & 2.47 & 0.25 & 16.84 & 15.1 & 23.07 & 22.33 & 0.13 \\
\hline $1256+2910$ & 3.48 & 3.29 & 0.37 & 16.14 & 10.4 & 22.96 & 21.66 & 0.11 \\
\hline $1256+2823$ & 3.03 & 3.03 & 0.36 & 15.94 & 12.7 & 22.84 & 21.72 & 0.15 \\
\hline $1256+2754$ & 4.22 & 3.82 & 0.47 & 15.39 & 7.6 & 22.56 & 20.74 & 0.14 \\
\hline $1256+2722$ & 2.65 & 2.68 & 0.33 & 17.26 & 7.1 & 22.36 & 21.29 & 0.08 \\
\hline $1257+2808$ & 3.14 & 3.09 & 0.45 & 16.29 & 5.2 & 22.23 & 20.73 & 0.13 \\
\hline $1258+2754$ & 3.12 & 3.14 & 0.38 & 15.95 & 10.7 & 22.76 & 21.55 & 0.11 \\
\hline $1259+2934$ & - & - & - & - & - & - & - & - \\
\hline $1259+3011$ & 4.42 & 3.73 & 0.60 & 16.30 & 8.9 & 22.15 & 20.00 & 0.03 \\
\hline $1259+2755$ & 4.12 & 3.74 & 0.46 & 15.47 & 11.5 & 22.50 & 20.61 & 0.12 \\
\hline $1300+2907$ & 4.28 & 3.82 & 0.54 & 17.30 & 5.2 & 22.34 & 20.89 & 0.05 \\
\hline $1301+2904$ & 3.35 & 3.32 & 0.31 & 15.70 & 12.3 & 22.98 & 21.95 & 0.17 \\
\hline $1302+2853$ & 3.09 & 3.22 & 0.43 & 16.39 & 7.3 & 22.37 & 20.89 & 0.05 \\
\hline $1302+3032$ & 3.20 & 3.26 & 0.48 & 16.70 & 8.5 & 22.29 & 20.67 & 0.10 \\
\hline $1303+2908$ & 2.21 & 2.25 & 0.24 & 16.94 & 9.9 & 22.94 & 22.04 & 0.22 \\
\hline $1304+2808$ & 3.20 & 3.10 & 0.42 & 16.00 & 13.2 & 23.02 & 21.89 & 0.10 \\
\hline $1304+2830$ & 2.49 & 2.43 & 0.31 & 18.66 & 2.1 & 22.39 & 21.14 & 0.01 \\
\hline $1304+2907$ & 3.06 & 2.95 & 0.33 & 15.28 & 10.9 & 23.21 & 22.37 & 0.41 \\
\hline $1304+2818$ & 2.57 & 2.57 & 0.26 & 15.86 & 9.7 & 22.71 & 21.82 & 0.06 \\
\hline $1306+2938$ & 3.55 & 3.49 & 0.41 & 15.44 & 8.2 & 22.11 & 20.43 & 0.15 \\
\hline $1306+3111$ & 2.53 & 2.56 & 0.33 & 16.37 & 4.4 & 22.29 & 20.97 & 0.09 \\
\hline $1307+2910$ & 3.44 & 3.37 & 0.30 & 14.31 & 26.2 & 23.17 & 22.25 & 0.11 \\
\hline $1308+2958$ & 2.37 & 2.42 & 0.25 & 15.40 & 14.6 & 22.98 & 22.32 & 0.10 \\
\hline $1308+2950$ & 2.90 & 2.94 & 0.34 & 15.03 & 27.5 & 23.16 & 22.43 & 0.03 \\
\hline
\end{tabular}


Table 3. continued

\begin{tabular}{|c|c|c|c|c|c|c|c|c|}
\hline $\begin{array}{l}\text { UCM name } \\
(1)\end{array}$ & $\begin{array}{l}c_{31} \\
(2) \\
\end{array}$ & $\begin{array}{l}c_{42} \\
(3)\end{array}$ & $\begin{array}{l}c_{\text {in }} \\
(4)\end{array}$ & $\begin{array}{c}b_{24.5} \\
(5) \\
\end{array}$ & $\begin{array}{c}r_{24.5} \\
(6)\end{array}$ & $\begin{array}{c}<\mu_{24.5}> \\
(7)\end{array}$ & $\begin{array}{c}<\mu_{\mathrm{e}}> \\
(8)\end{array}$ & $\begin{array}{c}A \\
(9) \\
\end{array}$ \\
\hline $1310+3027$ & 3.73 & 3.54 & 0.44 & 16.72 & 8.4 & 22.66 & 21.33 & 0.07 \\
\hline $1312+3040$ & 3.89 & 3.56 & 0.54 & 15.64 & 9.8 & 22.46 & 20.88 & 0.06 \\
\hline $1312+2954$ & 2.84 & 2.85 & 0.29 & 16.20 & 10.9 & 22.79 & 21.82 & 0.05 \\
\hline $1313+2938$ & 3.58 & 3.38 & 0.53 & 16.81 & 6.6 & 21.84 & 19.85 & 0.10 \\
\hline $1314+2827$ & 3.43 & 3.39 & 0.58 & 16.24 & 7.4 & 22.56 & 20.37 & 0.06 \\
\hline $1320+2727$ & 2.81 & 2.94 & 0.46 & 17.50 & 4.6 & 22.39 & 20.73 & 0.02 \\
\hline $1324+2926$ & 5.07 & 4.53 & 0.49 & 17.99 & 2.4 & 22.39 & 21.20 & 0.07 \\
\hline $1324+2651$ & 8.03 & 5.27 & 0.61 & 15.18 & 11.6 & 22.11 & 19.35 & 0.30 \\
\hline $1331+2900$ & 3.59 & 3.44 & 0.40 & 19.10 & 3.1 & 22.60 & 21.54 & 0.11 \\
\hline $1428+2727$ & 3.01 & 3.00 & 0.45 & 14.88 & 8.2 & 22.04 & 20.36 & 0.23 \\
\hline $1429+2645$ & 3.37 & 3.25 & 0.39 & 17.88 & 5.1 & 22.76 & 21.63 & 0.02 \\
\hline $1430+2947$ & 5.47 & 4.44 & 0.51 & 16.80 & 8.3 & 22.82 & 21.55 & 0.02 \\
\hline $1431+2854$ & 3.26 & 3.16 & 0.45 & 15.59 & 13.0 & 22.49 & 21.01 & 0.14 \\
\hline $1431+2702$ & 2.69 & 2.78 & 0.40 & 17.07 & 6.9 & 22.30 & 20.98 & 0.06 \\
\hline $1431+2947$ & 4.49 & 4.14 & 0.35 & 18.07 & 4.8 & 23.26 & 22.89 & 0.07 \\
\hline $1431+2814$ & 3.28 & 3.06 & 0.50 & 17.01 & 10.1 & 22.49 & 21.03 & 0.06 \\
\hline $1432+2645$ & 3.39 & 3.62 & 0.38 & 15.54 & 19.5 & 23.01 & 21.82 & 0.09 \\
\hline $1440+2521 \mathrm{~S}$ & 3.27 & 3.21 & 0.45 & 17.03 & 9.7 & 22.79 & 21.64 & 0.32 \\
\hline $1440+2511$ & 3.64 & 3.63 & 0.39 & 16.89 & 13.0 & 23.41 & 22.97 & 0.06 \\
\hline $1440+2521 \mathrm{~N}$ & 3.02 & 3.11 & 0.42 & 16.77 & 11.1 & 22.87 & 21.68 & 0.08 \\
\hline $1442+2845$ & 3.20 & 3.23 & 0.44 & 15.42 & 5.0 & 22.60 & 21.17 & 0.06 \\
\hline $1443+2714$ & 2.91 & 2.91 & 0.41 & 15.85 & 10.5 & 22.62 & 21.06 & 0.04 \\
\hline $1443+2844$ & 2.10 & 2.09 & 0.30 & 15.71 & 11.9 & 22.14 & 21.08 & 0.12 \\
\hline $1443+2548$ & 2.63 & 2.69 & 0.38 & 15.78 & 12.8 & 22.35 & 21.20 & 0.10 \\
\hline $1444+2923$ & 5.97 & 4.50 & 0.53 & 16.88 & 8.7 & 23.13 & 22.36 & 0.07 \\
\hline $1452+2754$ & 3.42 & 3.01 & 0.46 & 16.42 & 11.8 & 22.51 & 21.40 & 0.05 \\
\hline $1506+1922$ & 4.30 & 3.98 & 0.44 & 16.14 & 9.1 & 22.85 & 21.68 & 0.04 \\
\hline $1513+2012$ & 3.69 & 3.63 & 0.47 & 16.04 & 15.3 & 22.44 & 20.74 & 0.23 \\
\hline $1537+2506 \mathrm{~N}$ & 4.20 & 4.11 & 0.39 & 15.26 & 12.2 & 22.63 & 21.23 & 0.13 \\
\hline $1537+2506 \mathrm{~S}$ & 3.57 & 3.43 & 0.43 & 16.24 & 7.9 & 22.47 & 20.77 & 0.24 \\
\hline $1557+1423$ & 2.90 & 2.85 & 0.41 & 16.81 & 7.7 & 22.67 & 21.43 & 0.11 \\
\hline $1612+1308$ & 2.45 & 2.31 & 0.23 & 18.57 & 1.4 & 22.88 & 22.07 & 0.03 \\
\hline $1646+2725$ & 3.03 & 3.15 & 0.35 & 18.48 & 6.2 & 22.85 & 22.19 & 0.17 \\
\hline $1647+2950$ & 2.77 & 2.88 & 0.32 & 15.51 & 11.5 & 22.41 & 21.21 & 0.08 \\
\hline $1647+2729$ & 2.59 & 2.53 & 0.44 & 16.02 & 13.6 & 22.36 & 20.99 & 0.41 \\
\hline $1647+2727$ & 1.99 & 1.83 & 0.45 & 16.12 & 14.0 & 22.44 & 20.63 & 0.03 \\
\hline $1648+2855$ & 2.89 & 3.01 & 0.49 & 15.56 & 9.7 & 21.91 & 20.32 & 0.22 \\
\hline $1653+2644$ & 5.65 & 4.66 & 0.60 & 14.94 & 19.5 & 22.24 & 20.02 & 0.21 \\
\hline $1654+2812$ & 2.89 & 2.95 & 0.19 & 18.47 & 7.0 & 23.20 & 22.69 & 0.14 \\
\hline $1655+2755$ & 2.87 & 2.93 & 0.29 & 15.90 & 19.3 & 23.24 & 22.61 & 0.06 \\
\hline $1656+2744$ & 3.23 & 3.17 & 0.38 & 17.52 & 6.3 & 22.84 & 21.73 & 0.06 \\
\hline $1657+2901$ & 2.42 & 2.44 & 0.28 & 17.20 & 6.7 & 22.12 & 20.89 & 0.03 \\
\hline $1659+2928$ & 5.75 & 4.70 & 0.60 & 16.10 & 13.6 & 22.46 & 20.91 & 0.06 \\
\hline $1701+3131$ & 5.53 & 4.53 & 0.72 & 15.41 & 19.9 & 22.48 & 19.87 & 0.41 \\
\hline $2238+2308$ & 3.49 & 3.24 & 0.47 & 14.82 & 16.1 & 22.74 & 20.97 & 0.13 \\
\hline $2239+1959$ & 4.21 & 3.85 & 0.58 & 14.97 & 13.7 & 22.19 & 20.33 & 0.13 \\
\hline $2249+2149$ & 3.30 & 3.23 & 0.38 & 16.09 & 21.3 & 23.04 & 21.91 & 0.06 \\
\hline $2250+2427$ & 4.44 & 3.91 & 0.60 & 15.42 & 20.0 & 22.14 & 20.22 & 0.22 \\
\hline
\end{tabular}


Table 3. continued

\begin{tabular}{|c|c|c|c|c|c|c|c|c|}
\hline $\begin{array}{l}\text { UCM name } \\
\text { (1) }\end{array}$ & $\begin{array}{l}c_{31} \\
(2)\end{array}$ & $\begin{array}{l}c_{42} \\
(3)\end{array}$ & $\begin{array}{l}c_{\text {in }} \\
(4)\end{array}$ & $\begin{array}{c}b_{24.5} \\
(5)\end{array}$ & $\begin{array}{c}r_{24.5} \\
(6)\end{array}$ & $\begin{array}{c}\mu_{24.5}> \\
(7)\end{array}$ & $\begin{array}{c}<\mu_{\mathrm{e}}> \\
(8)\end{array}$ & $\begin{array}{c}A \\
(9)\end{array}$ \\
\hline $2251+2352$ & 2.58 & 2.54 & 0.35 & 16.41 & 5.8 & 22.03 & 20.63 & 0.12 \\
\hline $2253+2219$ & 2.88 & 2.87 & 0.48 & 16.15 & 8.0 & 22.11 & 20.51 & 0.08 \\
\hline $2255+1930 \mathrm{~S}$ & 2.64 & 2.60 & 0.46 & 16.16 & 4.7 & 21.75 & 19.98 & - \\
\hline $2255+1930 \mathrm{~N}$ & 3.45 & 3.35 & 0.53 & 15.90 & 9.4 & 22.44 & 20.82 & 0.04 \\
\hline $2255+1926$ & 3.39 & 3.22 & 0.24 & 17.15 & 7.0 & 23.16 & 22.45 & 0.15 \\
\hline $2255+1654$ & 2.54 & 2.60 & 0.30 & 16.85 & 18.1 & 23.04 & 22.41 & 0.11 \\
\hline $2256+2001$ & 2.28 & 2.29 & 0.18 & 15.86 & 14.8 & 23.45 & 23.02 & 0.07 \\
\hline $2257+2438$ & 4.15 & 3.77 & 0.73 & 16.13 & 7.3 & 21.65 & 18.74 & 0.06 \\
\hline $2257+1606$ & 4.07 & 3.92 & 0.58 & 16.50 & 7.8 & 22.36 & 20.06 & 0.06 \\
\hline $2258+1920$ & 2.65 & 2.70 & 0.34 & 15.72 & 7.5 & 22.19 & 20.84 & 0.11 \\
\hline $2300+2015$ & 3.18 & 3.23 & 0.34 & 16.79 & 8.6 & 22.64 & 21.48 & 0.21 \\
\hline $2302+2053 \mathrm{~W}$ & 3.48 & 3.49 & 0.47 & 18.18 & 5.1 & 22.48 & 21.23 & - \\
\hline $2302+2053 \mathrm{E}$ & 4.16 & 4.01 & 0.54 & 15.81 & 15.4 & 22.61 & 21.63 & 0.15 \\
\hline $2303+1856$ & 3.30 & 3.22 & 0.45 & 15.99 & 12.7 & 22.56 & 21.03 & 0.04 \\
\hline $2303+1702$ & 2.74 & 2.76 & 0.19 & 17.74 & 9.5 & 23.00 & 22.28 & 0.05 \\
\hline $2304+1640$ & 2.78 & 2.82 & 0.35 & 17.95 & 2.8 & 22.69 & 21.71 & 0.11 \\
\hline $2304+1621$ & 3.71 & 3.52 & 0.47 & 17.27 & 8.5 & 22.77 & 21.50 & 0.07 \\
\hline $2307+1947$ & 3.37 & 3.37 & 0.44 & 16.78 & 8.0 & 22.66 & 21.48 & 0.04 \\
\hline $2310+1800$ & 3.83 & 3.50 & 0.32 & 16.90 & 8.6 & 22.77 & 21.54 & 0.10 \\
\hline $2312+2204$ & 4.53 & 4.04 & 0.54 & 17.35 & 7.4 & 22.66 & 21.21 & 0.07 \\
\hline $2313+1841$ & 3.20 & 3.04 & 0.37 & 17.22 & 8.4 & 22.88 & 21.73 & 0.02 \\
\hline $2313+2517$ & 4.67 & 4.27 & 0.52 & 15.10 & 17.8 & 22.72 & 20.90 & 0.12 \\
\hline $2315+1923$ & 3.63 & 3.59 & 0.44 & 17.58 & 7.2 & 22.66 & 21.49 & 0.03 \\
\hline $2316+2457$ & 4.26 & 4.14 & 0.44 & 14.48 & 18.9 & 22.37 & 20.45 & 0.17 \\
\hline $2316+2459$ & 2.70 & 2.81 & 0.24 & 16.24 & 11.2 & 23.06 & 22.41 & 0.16 \\
\hline $2316+2028$ & 4.15 & 3.90 & 0.56 & 17.08 & 5.7 & 22.35 & 20.71 & 0.02 \\
\hline $2317+2356$ & 3.50 & 3.42 & 0.44 & 13.97 & 26.9 & 22.28 & 20.82 & 0.13 \\
\hline $2319+2234$ & 4.49 & 4.10 & 0.37 & 16.83 & 8.8 & 22.22 & 20.99 & 0.06 \\
\hline $2319+2243$ & 5.50 & 4.61 & 0.52 & 15.98 & 13.9 & 22.69 & 21.34 & 0.03 \\
\hline $2320+2428$ & 3.54 & 3.41 & 0.52 & 15.73 & 24.6 & 22.67 & 21.45 & 0.01 \\
\hline $2321+2149$ & 2.74 & 2.85 & 0.23 & 16.71 & 9.0 & 22.60 & 21.52 & 0.11 \\
\hline $2321+2506$ & 2.68 & 2.63 & 0.39 & 15.83 & 15.6 & 22.54 & 21.39 & 0.08 \\
\hline $2322+2218$ & 2.83 & 2.85 & 0.35 & 17.83 & 5.0 & 22.69 & 21.62 & 0.05 \\
\hline $2324+2448$ & 3.80 & 3.66 & 0.43 & 13.58 & 19.7 & 22.62 & 21.33 & 0.21 \\
\hline $2325+2318$ & 3.19 & 3.21 & 0.58 & 13.32 & 12.1 & 22.04 & 19.95 & 0.29 \\
\hline $2325+2208$ & 2.00 & 1.98 & 0.22 & 12.96 & 21.5 & 22.79 & 22.00 & 0.21 \\
\hline $2326+2435$ & 2.91 & 2.91 & 0.40 & 16.59 & 8.5 & 22.85 & 21.89 & 0.09 \\
\hline $2327+2515 \mathrm{~N}$ & 3.20 & 3.08 & 0.54 & 15.43 & 11.3 & 22.61 & 19.63 & - \\
\hline $2327+2515 \mathrm{~S}$ & 3.77 & 3.42 & 0.53 & 15.61 & 9.1 & 22.60 & 20.28 & 0.23 \\
\hline $2329+2427$ & 3.59 & 3.56 & 0.45 & 16.02 & 11.6 & 22.77 & 21.77 & 0.04 \\
\hline $2329+2500$ & 8.33 & 5.47 & 0.65 & 16.43 & 12.1 & 22.91 & 20.43 & 0.03 \\
\hline $2329+2512$ & 5.09 & 4.36 & 0.58 & 16.81 & 3.5 & 22.38 & 20.93 & 0.01 \\
\hline $2331+2214$ & 4.09 & 3.92 & 0.47 & 17.93 & 8.0 & 23.16 & 22.25 & 0.07 \\
\hline $2333+2248$ & 3.42 & 3.28 & 0.38 & 17.18 & 17.2 & 22.98 & 21.88 & 0.19 \\
\hline $2333+2359$ & 4.13 & 3.76 & 0.44 & 17.96 & 6.2 & 23.01 & 21.89 & 0.06 \\
\hline $2348+2407$ & 3.40 & 3.36 & 0.37 & 18.20 & 4.8 & 22.69 & 21.48 & 0.01 \\
\hline $2351+2321$ & 3.04 & 3.01 & 0.42 & 17.82 & 4.0 & 22.57 & 21.20 & 0.07 \\
\hline
\end{tabular}

(1) UCM name. (2) $c_{31}$ index as defined by de Vaucouleurs (1977). Mean error is $\sim 1 \%$. (3) $c_{42}$ index calculated after Kent (1985). Mean error is $\sim 2 \%$. (4) $c_{\text {in }}$ as defined by Doi et al. (1993). Mean error is $\sim 6 \%$. (5) Johnson $B$ magnitude measured inside the $24.5 \mathrm{mag} \operatorname{arcsec}^{-2}$ isophote. Mean error is $0.06^{\mathrm{m}}$. (6) Radius of the $24.5 \mathrm{mag} \operatorname{arcsec}^{-2}$ isophote in kpc. Mean error is $\sim 11 \%$. (7) Mean surface brightness inside the $24.5 \mathrm{mag} \operatorname{arcsec}^{-2}$ isophote. Mean error is $\sim 0.06^{\mathrm{m}}$. (8) Mean effective surface brightness. Mean error is $\sim 0.02^{\mathrm{m}}$. (9) Asymmetry coefficient calculated as in Abraham et al. (1996). Mean error is $\sim 2 \%$. 


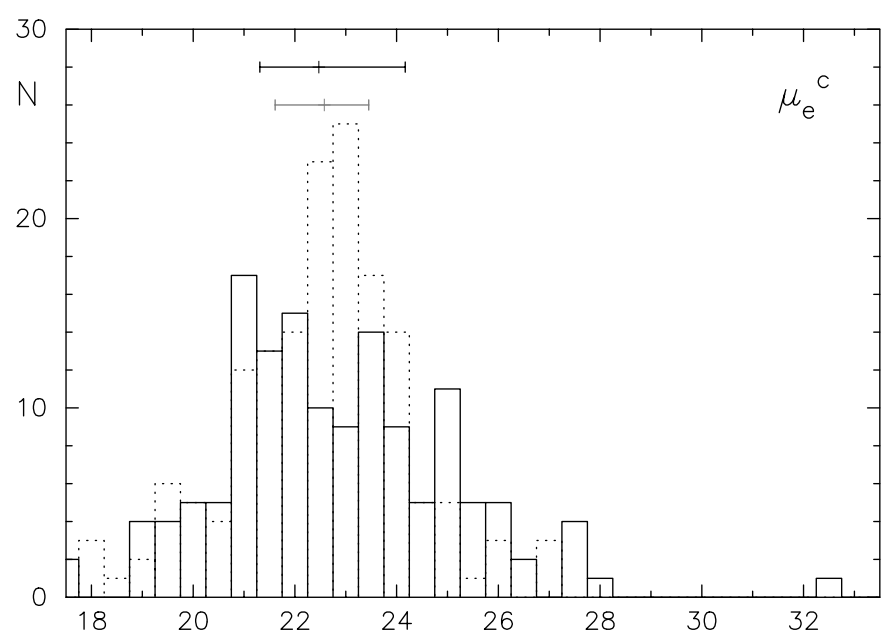

Fig. 2. Histogram of the effective bulge surface brightness $\mu_{\mathrm{e}}^{\mathrm{c}}$ corrected for Galactic extinction

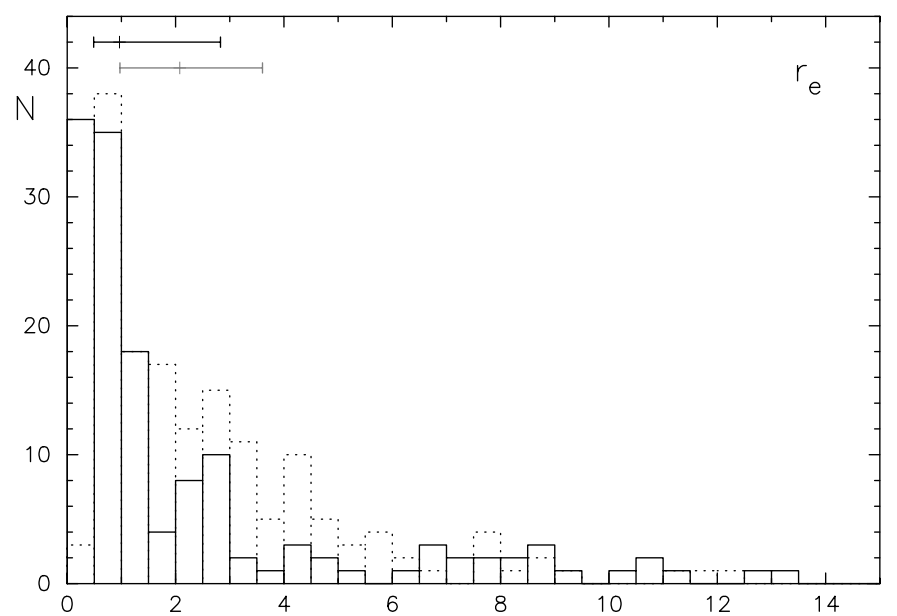

Fig. 3. Histogram of the effective radius of the bulge component in kpc

$\mu_{0}^{\mathrm{c}}=21-22 \mathrm{mag} \operatorname{arcsec}^{-2}$, with the average in $21.1 \pm$ $1.1 \mathrm{mag} \operatorname{arcsec}^{-2}\left(21.2 \pm 1.1 \mathrm{mag} \operatorname{arcsec}^{-2}\right.$ for the low- $B / D$ subsample). The narrow range of $\mu_{0}^{\mathrm{c}}$ seems to support the existence of a universal central surface brightness for the disk, as proposed for normal spirals by Freeman (1970) and confirmed by other authors (i.e., Boroson 1981; Simien \& de Vaucouleurs 1986), although other works in the literature present samples of galaxies with a wider spread in $\mu_{0}^{\mathrm{c}}$ (see, for example, McGaugh et al. 1995 or Beijersbergen et al. 1999). Our $\mu_{0}^{\mathrm{c}}$ value is $\sim 0.5^{\mathrm{m}}$ brighter than the Freeman central surface brightness. Therefore, the UCM sample of star-forming galaxies appears to have brighter disks than those of normal spirals; this fact is probably related to the higher star-formation activity. Scale lengths are dominated by disks smaller than $4 \mathrm{kpc}(68 \%$ of the total number of galaxies fitted), with mean $3.6 \pm 2.6 \mathrm{kpc}$ (the same for the low- $B / D$ subsample). This value is higher than that found by Chitre et al. (1999) for a sample of starburst galaxies in the Markarian sample $\left(d_{\mathrm{L}}^{\mathrm{c}}<3 \mathrm{kpc}\right)$, very similar to the averaged value found by Vennik et al. (2000) for a sample of emission-line galaxies $\left(d_{\mathrm{L}}^{\mathrm{c}} \sim 2.7 \mathrm{kpc}\right)$,

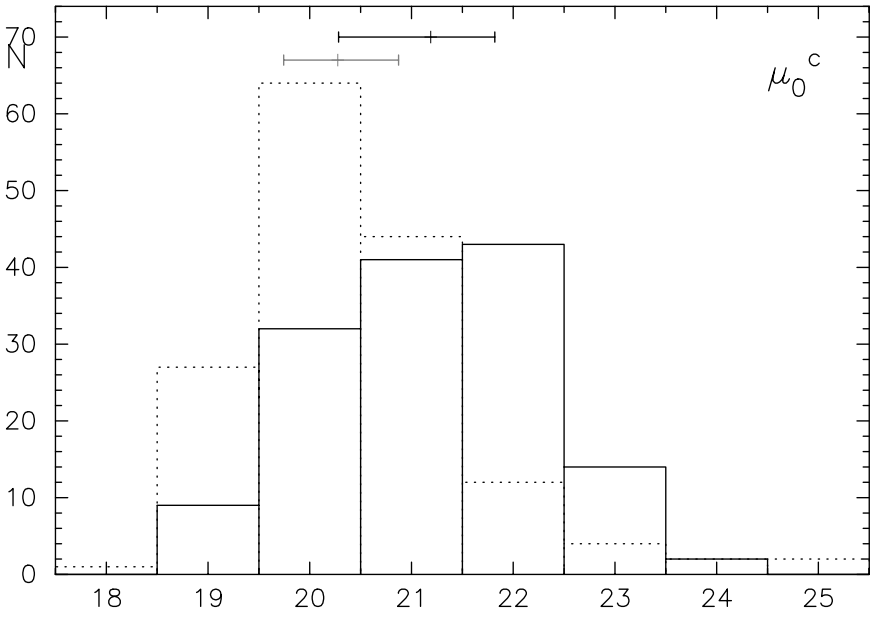

Fig. 4. Histogram of the characteristic surface brightness of the disk $\mu_{0}^{\mathrm{c}}$ corrected for Galactic extinction and inclination

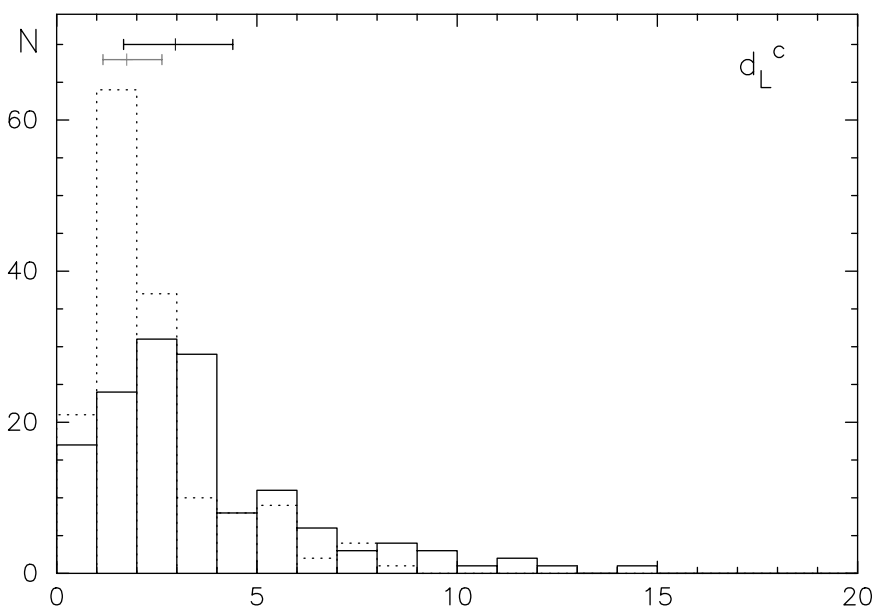

Fig. 5. Histogram of the exponential scale length of the disk $d_{\mathrm{L}}^{\mathrm{c}}$ measured in $\mathrm{kpc}$

although they only fit an exponential to the outer parts of the profiles. Our value is lower than the one found by de Jong (1996a) for normal edge-on spirals $\left(d_{\mathrm{L}}^{\mathrm{c}} \sim 8 \mathrm{kpc}\right)$ - they argue that their selection biases against galaxies with low surface brightness and short scale lengths are large -. Other works (for example, Boroson 1981; Kent 1985; Bothun et al. 1989; Andredakis \& Sanders 1994) agree in placing our galaxies in the zone of short disk spirals, though one should be cautious against comparing scale lengths from different authors due to the subjective nature of disk parameters (Knapen \& van der Kruit 1991 find discrepancies up to a factor of two in the scale lengths calculated from several authors).

All of the above values are very similar to those found by Vitores et al. (1996b) and place the UCM sample of galaxies in the zone of the late-type spirals, with small bulges and not very extended disks (Freeman 1970). Three remarks are interesting when comparing both sets of data. First, in the Gunn $r$ decomposition a lower bulge scale cutoff was observed (at $r_{\mathrm{e}}=0.5 \mathrm{kpc}$ ); this is not present in the $B$ band study. A possible explanation is the different 


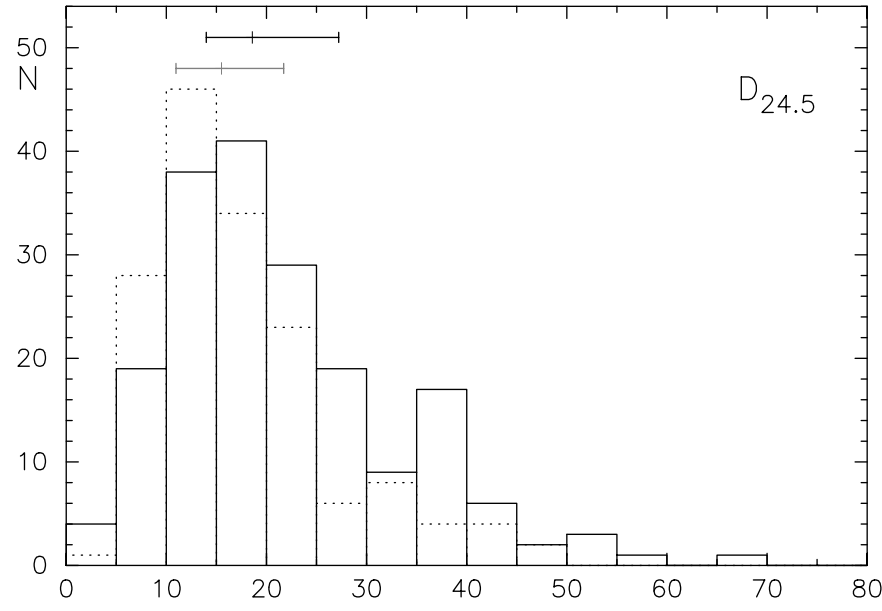

Fig. 6. Histogram of the diameter of the $24.5 \mathrm{mag} \mathrm{arcsec}^{-2}$ isophote $D_{24.5}$ in $\mathrm{kpc}$

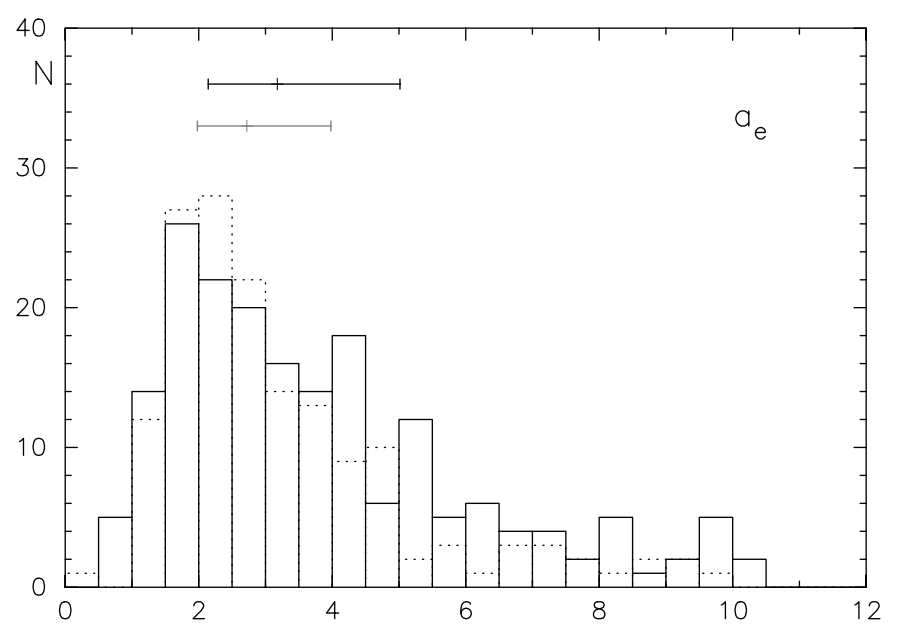

Fig. 7. Histogram of effective radius $a_{\mathrm{e}}$ in $\mathrm{kpc}$

handling performed with the seeing that allows the $B$ bulges to be smaller but brighter (seeing correction smoothes the profile; this was not the case with the Gunn $r$ bulge-disk decomposition, where the seeing effect was not taken into account directly), but this does not seem to cope with the whole difference. Second, both bands present a preference for disk scales around 2-3 kpc (larger disks in the blue band); very short disk scales and large ones are less frequent. Third, the difference between the surface brightness levels of the bulge and disk are of the order of the mean colour, around $0.5^{\mathrm{m}}$, as expected according to the averaged $B-r$ colour found in Fukugita et al. (1995).

\subsection{Geometric parameters}

In order to typify the size of the UCM galaxies, the histograms representing the diameter of the 24.5 mag arcsec ${ }^{-2}$ isophote $D_{24.5}$ and the effective radius $a_{\mathrm{e}}$ (both in kpc) have been plotted in Figs. 6 and 7 . The averaged diameter of the UCM objects is $22 \pm$ $12 \mathrm{kpc}$. Comparison with the red data has been established

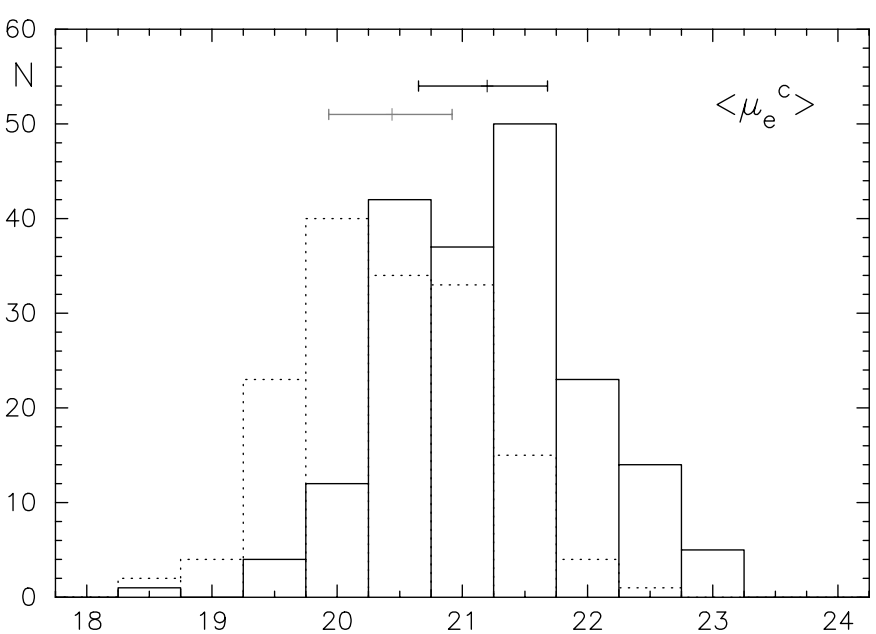

Fig. 8. Histogram of the mean surface brightness inside the effective aperture $<\mu_{\mathrm{e}}^{\mathrm{c}}>$ corrected for Galactic extinction

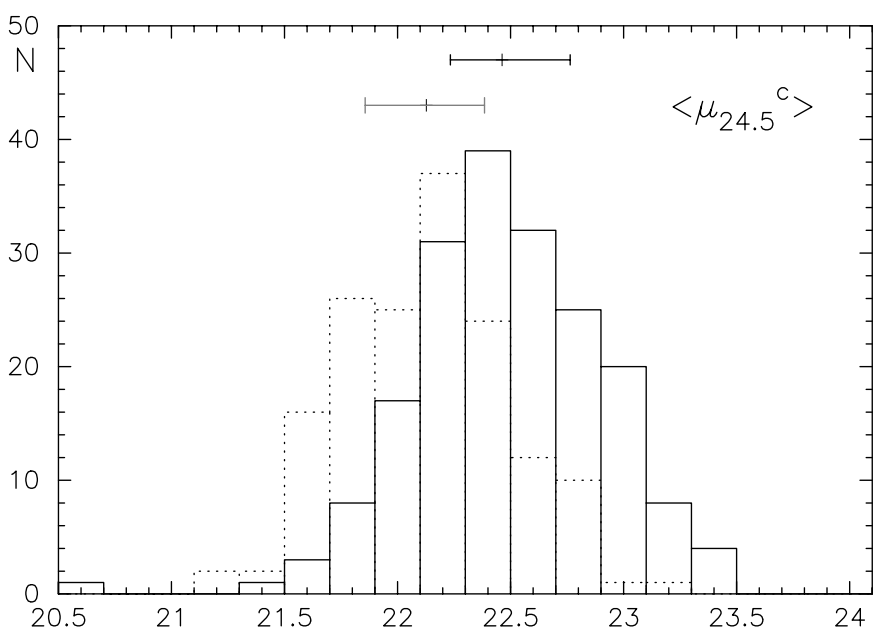

Fig. 9. Histogram of the mean surface brightness inside the 24.5 isophote $<\mu_{24.5}^{\mathrm{c}}>$ corrected for Galactic extinction

through the plot of the diameter of the 24 mag $\operatorname{arcsec}^{-2}$ Gunn $r$ isophote (that will be nearer to the 24.5 blue isophote than the corresponding red one, assuming a mean colour $B-r \sim 0.5$ ). The mean effective radius $a_{\mathrm{e}}$ is $3.8 \pm 2.3 \mathrm{kpc}$; this reflects the high degree of spatial luminosity concentration of our objects, most of them being starburst nuclei with a large emission arising from the center of the galaxy. Tentatively, UCM galaxies seem to be more extended in the blue band than in the red one (they show larger effective radius and diameters in $B$ ).

Finally, we plot in Figs. 8 and 9 the mean effective and isophote 24.5 surface brightnesses in order to characterize the whole galaxy luminosity distribution. UCM objects show $\left\langle\mu_{\mathrm{e}}^{\mathrm{c}}\right\rangle=21.2 \pm 0.9$ and $\left\langle\mu_{24.5}^{\mathrm{c}}\right\rangle=22.5 \pm 0.4$ (both in mag arcsec ${ }^{-2}$ ), common value for normal galaxies (Doi et al. 1993). The difference between the Gunn $r$ and the Johnson $B$ values $\left(\sim 0.5^{\mathrm{m}}\right)$ is a common $B-r$ colour for spirals (Fukugita et al. 1995). 


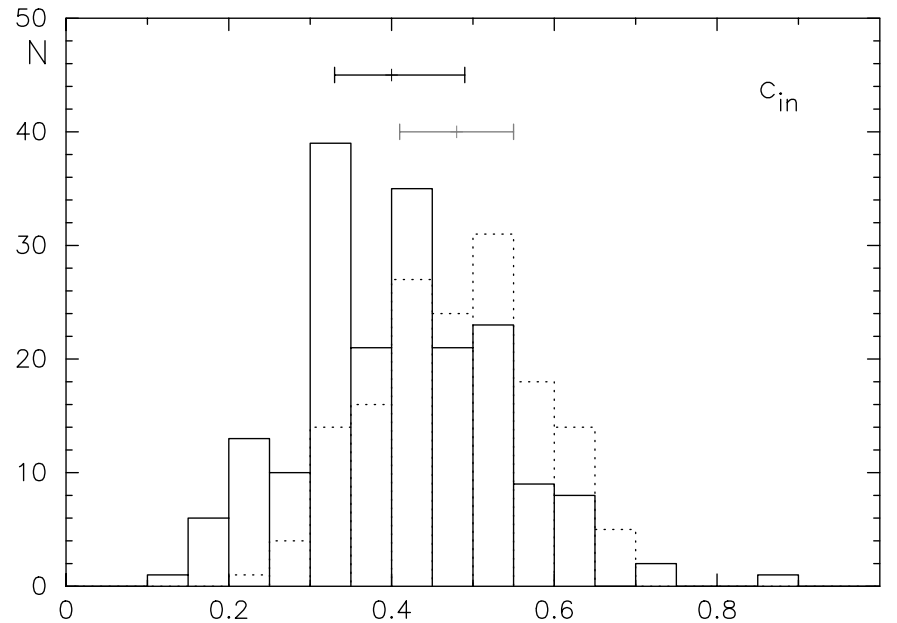

Fig. 10. Histogram of the concentration index $c_{\text {in }}$ of the UCM sample

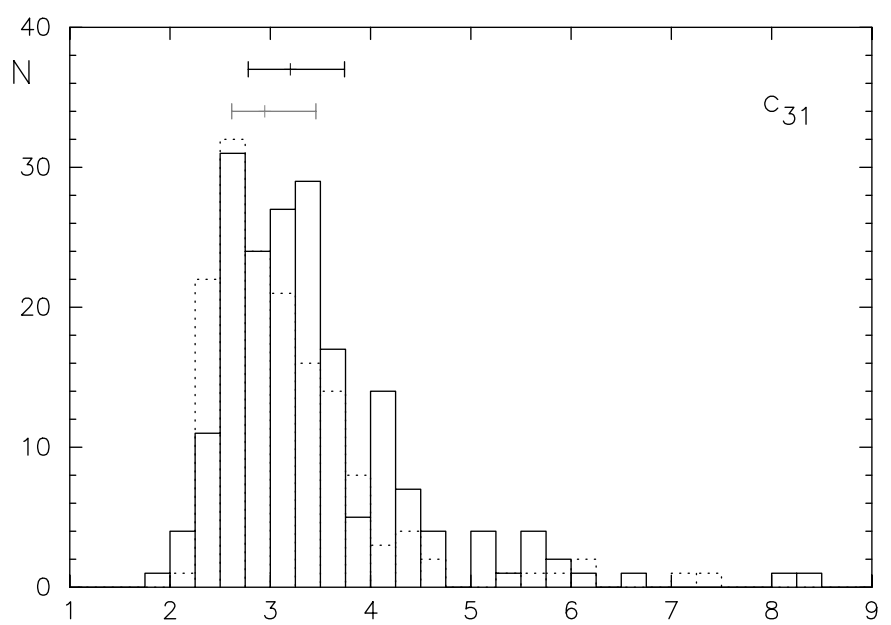

Fig. 11. Histogram of the concentration index $c_{31}$ of the UCM sample

\subsection{Concentration indices and asymmetry coefficient}

In the next 3 figures, labelled 10, 11 and 12, histograms of the concentration indices are shown. Mean values are $c_{\text {in }}=0.41 \pm 0.12, c_{31}=3.4 \pm 1.0$ and $c_{42}=3.3 \pm 0.6$. All of them are common values for spiral galaxies, corresponding approximately to a Hubble type of Sb (Doi et al. 1993; Gavazzi et al. 1990; Kent 1985; respectively for each concentration index). These values are higher than those measured in the Gunn $r$ images. The $B$ luminosity seems to be more concentrated in the inner parts than the $r$ one, although galaxies are more extended.

Figure 13 depicts the histogram of the asymmetry coefficient for the UCM sample. The UCM sample is dominated by intermediately asymmetrical galaxies with mean $0.10 \pm 0.08$, lower than the value found by Bershady et al. (2000) for a sample of normal local galaxies; this could be due to a difference in the calculation of $A$ or because their sample is composed by bright, large objects which probably have many asymmetrical features. This is what we should expect for spirals which have a certain axis

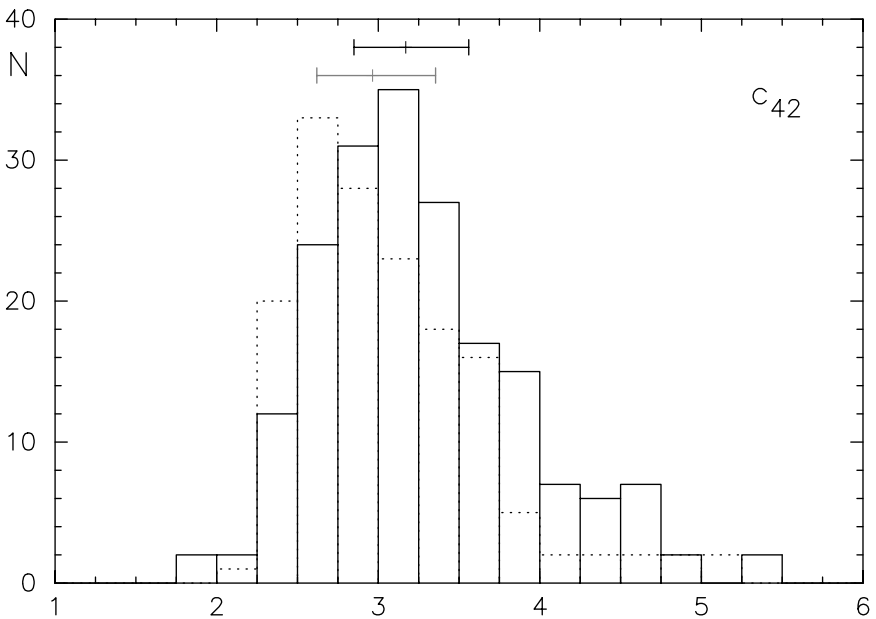

Fig. 12. Histogram of the concentration index $c_{42}$ of the UCM sample

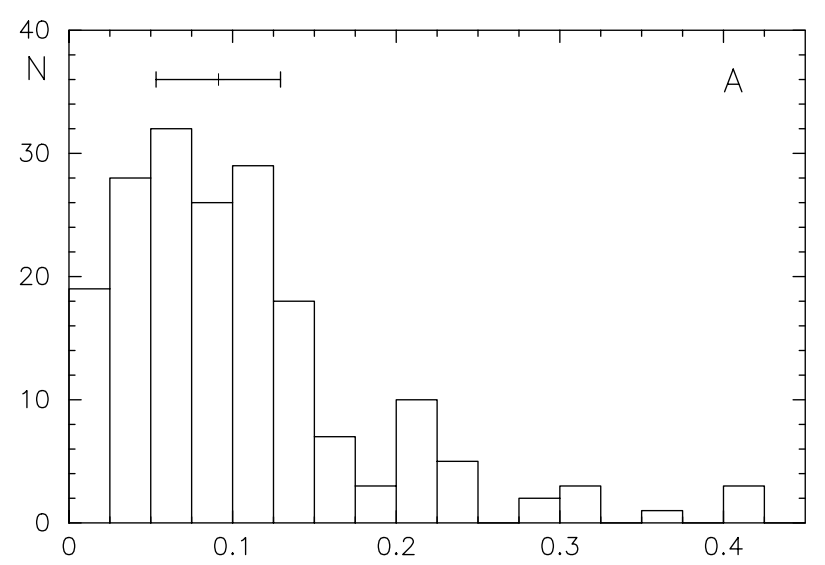

Fig. 13. Histogram of the asymmetry coefficient $A$ of the UCM sample calculated after Abraham et al. (1996)

symmetry although they present arms, bars or HII regions that enlarge the asymmetry coefficient. There is a lack of highly symmetrical objects, which correspond to elliptical galaxies, not present in our sample as it is composed by star-forming systems.

All the previous results have been summarized in Table 4 for a quick look, jointly with the Gunn $r$ statistics.

\subsection{Morphological classification}

A morphological classification of the UCM galaxies has been carried out using 5 different criteria. These criteria were already used by Vitores et al. (1996a) with the Gunn $r$ images, and are now applied to the Johnson $B$ data in order to compare the results obtained with different bandpasses. Besides, some galaxies not studied in Vitores et al. (1996a) have now been classified for the first time 

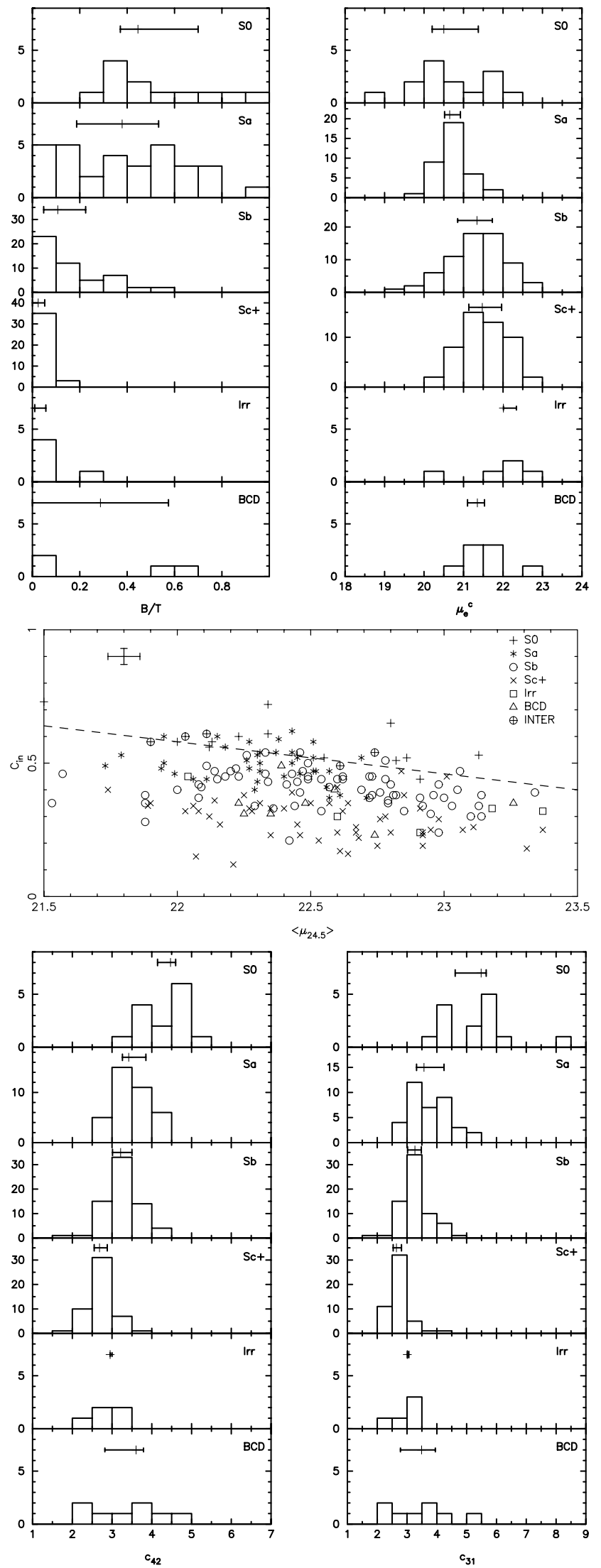

Fig. 14. Plots of the 5 criteria used to morphologically classify the UCM galaxies. Top panels show on the left the distribution of the $B / T$ ratio and on the right the mean effective surface brightness (corrected for Galactic extinction) according to the final Hubble type established for each galaxy. The middle panel shows the plot found in Doi et al. (1993) of concentration index $c_{\text {in }}$ versus isophote 24.5 mean surface brightness; the dashed line represents the segregation between late-type and early-type established by Doi et al. (1993). Lower panels are the histograms of the concentration indices $c_{42}$ (left) and $c_{31}$ (right) 
Table 4. Mean, median and standard deviation of the photometric parameters of the Johnson $B$ and Gunn $r$ (in brackets) images of the UCM Survey galaxies (scales are in kpc and surface brightnesses in mag $\operatorname{arcsec}^{-2}$ )

\begin{tabular}{|c|c|c|c|c|}
\hline Magnitudes & symbol & mean & st. dev. & median \\
\hline \multicolumn{5}{|l|}{ Magnitudes } \\
\hline apparent magnitude & $m_{B}$ & $16.1(15.5)$ & $1.1(1.0)$ & $16.1(15.5)$ \\
\hline absolute magnitude & $M_{B}$ & $-19.9(-20.5)$ & $1.1(1.1)$ & $-20.0(-20.6)$ \\
\hline \multicolumn{5}{|l|}{$\mathrm{B}+\mathrm{D}$ parameters } \\
\hline bulge-to-disk ratio & $B / D$ & $0.40(0.82)$ & $0.65(0.98)$ & $0.12(0.48)$ \\
\hline effective bulge surface brightness & $\mu_{\mathrm{e}}^{\mathrm{c}}$ & $22.8(22.6)$ & $2.3(1.7)$ & $22.5(22.6)$ \\
\hline effective radius of the bulge & $r_{\mathrm{e}}$ & $2.7(2.1)$ & $4.8(3.3)$ & $1.0(2.1)$ \\
\hline disk face-on central surface brightness & $\mu_{0}^{\mathrm{c}}$ & $21.1(20.3)$ & $1.1(1.1)$ & $21.2(20.3)$ \\
\hline exponential scale length of the disk & $d_{\mathrm{L}}^{\mathrm{c}}$ & $3.6(1.8)$ & $2.6(1.6)$ & $3.0(1.8)$ \\
\hline \multicolumn{5}{|l|}{ Geometric parameters } \\
\hline \multicolumn{5}{|l|}{ Mean photometric parameters } \\
\hline effective radius & $a_{\mathrm{e}}$ & $3.8(3.3)$ & $2.3(1.9)$ & $3.2(2.7)$ \\
\hline mean effective surface brightness & $<\mu_{\mathrm{e}}^{\mathrm{c}}>$ & $21.2(20.4)$ & $0.9(0.7)$ & $21.2(20.4)$ \\
\hline isophote $24.5 \mathrm{mag} \operatorname{arcsec}^{-2}$ mean surface brightness & $\left.<\mu_{24.5}^{\mathrm{c}}\right\rangle$ & $22.5(22.1)$ & $0.4(0.4)$ & $22.5(22.1)$ \\
\hline \multicolumn{5}{|l|}{ Concentration indices } \\
\hline concentration index $(\alpha=0.3)$ & $c_{\text {in }}$ & $0.41(0.48)$ & $0.12(0.10)$ & $0.40(0.48)$ \\
\hline concentration index & $c_{31}$ & $3.4(3.2)$ & $1.0(0.9)$ & $3.2(3.0)$ \\
\hline concentration index & $c_{42}$ & $3.3(3.1)$ & $0.6(0.6)$ & $3.2(3.0)$ \\
\hline $\begin{array}{l}\text { Asymmetry coefficient } \\
\text { asymmetry coefficient }\end{array}$ & $A$ & $0.10(-)$ & $0.08(-)$ & $0.09(-)$ \\
\hline
\end{tabular}

( $15 \%$ of the sample). We outline the main features of the classification criteria:

- the correlation between $B / T$ ratio and Hubble type. $B / T$ is defined as:

$B / T=\frac{1}{(B / D)^{-1}+1}$.

This correlation was studied by Kent (1985, Fig. 6) for a sample of bright galaxies in a red filter. It has been assumed that the behaviour of the correlation is very similar in the blue band;

- the dependence of the Hubble type on the position in the plane defined by the concentration index $c_{\mathrm{in}}(\alpha)$ and $\mu_{24.5}^{\mathrm{c}}$, first studied by Doi et al. (1993). These authors argue that this criterium is rather insensitive to the colour band;

- the correlation between the concentration index $c_{31}$ and the Hubble type, as studied by Gavazzi et al. (1990, Fig. 4b);

- the dependence of the morphological type on the concentration index $c_{42}$, established by Kent (1985, Fig. 11);

- the correlation between the mean surface brightness inside the effective isophote (corrected for Galactic extinction) and the Hubble type (Kent 1985, Fig. 13). A mean correction of $0.5^{\mathrm{m}}$ due to the different bandpasses used in both works has been applied.

Visual inspection of each image was also used for the classification.

In this work we utilized all these five criteria to classify the UCM galaxies in $\mathrm{S} 0, \mathrm{Sa}, \mathrm{Sb}, \mathrm{Sc}+$ (Sc type or later) and Irr galaxies plus the BCD type (these galaxies were classified using spectroscopic confirmation available in Gallego et al. 1996); some galaxies were very distorted due to interactions and are marked in the result table as an independent class. The final Hubble type was established as that in which most criteria agree. This method is not completely objective and constitutes the main reason for the discrepancy between the classification using the Gunn $r$ data and that performed in this paper with the Johnson $B$ images. Table 5 presents the final classification in both bands. Figure 14 shows the histograms and plots of the 5 criteria used in the classification; in these plots the general trend of each parameter with the Hubble type can be seen, although great scatter and overlap between the different types are also present. Mean values will be shown in Table 7.

Table 6 presents the number of UCM galaxies of each type in the Gunn $r$ and Johnson $B$ filters. A total number of 35 galaxies have been classified differently in the two bands, although the differences are always from one type to the contiguous (except in UCM 2316+2028). Based on the Johnson $B$ data, $65 \%$ of the whole sample is classified as $\mathrm{Sb}$ or later (61\% based on Gunn $r$ images). The percentage of barred galaxies is very similar in both bands (Johnson B 9\%, Gunn $r 8 \%$ ); most of them are late-type spirals (47\% are $\mathrm{Sb}$ galaxies and $35 \% \mathrm{Sc}+$ ). We have marked 6 clear interactions among the UCM galaxies $(3 \%)$, although there are more objects with tails or structures that could have been formed during an interaction. Seyfert 1 galaxies (6 objects) are all classified as S0, except one (UCM 0003+1955) that is very bright and could not be classified; Sy 2 galaxies have been classified as $\mathrm{Sa}$ 
$(1$ object $), \quad \mathrm{Sb} \quad(3$ objects $)$ and $\mathrm{Sc}+\left(\begin{array}{ll}3 & \text { objects }\end{array}\right)$. These results are consistent with the ones found in the literature (see, for example, Hunt \& Malkan 1999).

\section{Correlations between parameters}

We plot in Figs. 15 and 16 the relationships between absolute $B$ magnitude and the size of the galaxy (24.5 isophote diameter) and also between $M_{B}$ and the distribution of light (concentration index $c_{31}$ ). Information about morphological classification is also shown.

There is a tight correlation between $M_{B}$ and $D_{24.5}$. A least-square fit to our data leads to:

$\log D_{24.5}=(-2.65 \pm 0.16)+(-0.20 \pm 0.01) M_{B}$.

The slope is very similar to the value expected for a constant luminosity-area ratio $(\log D=C-0.2 M)$. Therefore, despite there is a great variety in morphological and spectroscopic types, a uniformity in the mean surface brightness is exhibited, as was also proved with the red data (Vitores et al. 1996b found a slope value of $-0.21 \pm 0.01)$. The fit gives a mean surface brightness value of $-13.3 \mathrm{mag} \mathrm{kpc}-2$.

In Fig. 16 a general trend between the concentration index $c_{31}$, the absolute $B$ magnitude $M_{B}$ and the morphological type is apparent. Early-type galaxies show medium-high magnitudes and high concentration indices. If we move downwards to the zone of low concentration index we find spirals, from Sa to late-type. Finally, BCDs have $c_{31}$ values typical for spirals but are fainter than normal galaxies.

Figure 17 shows the segregation in morphological type in a $c_{\text {in }}$ versus $A$ diagram. In this plot and the next, median values for the different morphological types are plotted with a black dot; ellipse semi-axes are the $\sigma$ of each parameter. There is a clear trend from left to right in decreasing Hubble type. S0 galaxies are placed in the high symmetry-high $c_{\text {in }}$ zone. BCDs also appear as highly symmetrical objects. On the other hand, irregulars are shown as highly asymmetrical objects in the top-left zone of the plot and interactive systems are located among the most asymmetrical galaxies. A trend can be also remarked in the spiral sequence: early-type galaxies are more symmetrical than late-type ones (due to the presence of more HII regions, for example).

Figure 14 showed that there is a clear correlation between the concentration indices and Hubble type. This trend is also observed with the asymmetry coefficient. Table 7 presents the mean values of the bulge-to-disk ratio, mean effective surface brightness, concentration indices and asymmetry coefficient of each Hubble type. The statistics of $A$ have been split into barred and non-barred objects; barred galaxies are more asymmetrical than nonbarred ones.

Figure 18 depicts the absolute $B$ magnitude of the UCM objects versus the mean effective surface brightness. Early-type galaxies appear as bright, high surface brightness objects. Late-type spirals have lower $\mu_{\mathrm{e}}$, although no

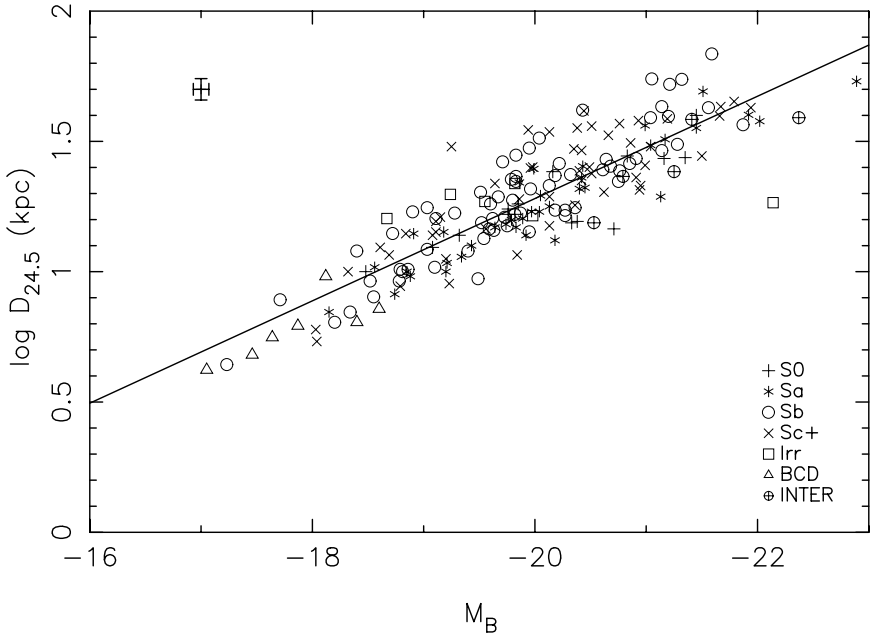

Fig. 15. Relationship between the size of the UCM galaxies represented by the diameter of the 24.5 isophote $D_{24.5}$ and the total $B$ luminosity of the object $M_{B}$. A least-square fit to the data is also plotted

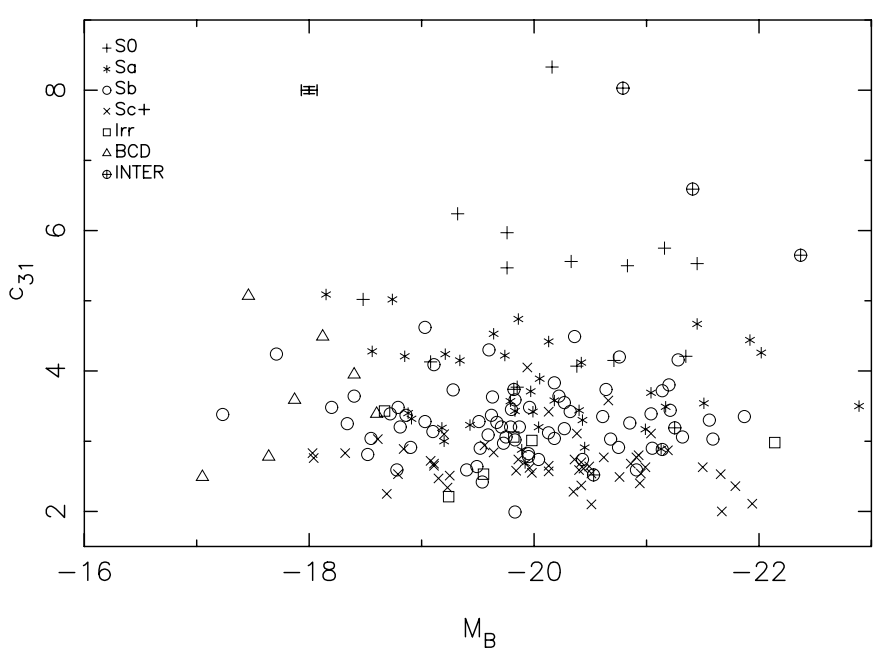

Fig. 16. Concentration index $c_{31}$ versus absolute magnitude $M_{B}$. Different symbols stand for distinct morphological types

significant difference in $M_{B}$ is present. BCDs are clearly segregated due to their faintness. Irregulars and interactive systems show also a distinctive surface brightness.

\section{Summary and conclusions}

We have carried out a morphological study of the UCM Survey galaxies based on Johnson $B$ imaging. This paper, jointly with Paper I (Pérez-González et al. 2000), have analyzed the main features of the UCM sample concerning integrated and surface photometry in the $B$ bandpass.

Paper I presented integrated apparent and absolute $B$ luminosities as well as isophote 24 mag $\operatorname{arcsec}^{-2}$ magnitudes. Effective radii and $B-r$ colours were also calculated in this first release. In the present paper we have outlined the main results concerning bulge-disk decomposition values, ellipticities, position angles, concentration 
Table 5. Morphological classification of the UCM sample of galaxies

\begin{tabular}{|c|c|c|c|c|c|c|c|c|}
\hline $\begin{array}{l}\text { UCM name } \\
(1)\end{array}$ & $\begin{array}{c}\operatorname{MpT}(B) \\
(2)\end{array}$ & $\begin{array}{c}\mathrm{MpT}(r) \\
(3)\end{array}$ & $\begin{array}{l}\text { UCM name } \\
\text { (1) }\end{array}$ & $\begin{array}{c}\operatorname{MpT}(B) \\
(2)\end{array}$ & $\begin{array}{c}\mathrm{MpT}(r) \\
(3)\end{array}$ & $\begin{array}{l}\text { UCM name } \\
(1)\end{array}$ & $\begin{array}{c}\operatorname{MpT}(B) \\
(2)\end{array}$ & $\begin{array}{c}\mathrm{MpT}(r) \\
(3)\end{array}$ \\
\hline $0000+2140$ & INTER & - & $0141+2220$ & $\mathrm{Sa}$ & $\mathrm{Sb}$ & $1314+2827$ & $\mathrm{Sa}$ & $\mathrm{Sa}$ \\
\hline $0003+2200$ & $\mathrm{Sc}+$ & $\mathrm{Sc}+$ & $0142+2137$ & $\mathrm{SBb}$ & $\mathrm{SBb}$ & $1320+2727$ & $\mathrm{Sb}$ & $\mathrm{Sb}$ \\
\hline $0003+2215$ & $\mathrm{Sc}+$ & - & $0144+2519$ & $\mathrm{SBc}+$ & $\mathrm{SBc}+(\mathrm{r})$ & $1324+2926$ & $\mathrm{BCD}$ & $\mathrm{BCD}$ \\
\hline $0003+1955$ & - & - & $0147+2309$ & $\mathrm{Sa}$ & $\mathrm{Sa}$ & $1324+2651$ & INTER & - \\
\hline $0005+1802$ & $\mathrm{Sb}$ & - & $0148+2124$ & $\mathrm{BCD}$ & $\mathrm{BCD}$ & $1331+2900$ & $\mathrm{BCD}$ & $\mathrm{BCD}$ \\
\hline $0006+2332$ & $\mathrm{Sb}$ & - & $0150+2032$ & $\mathrm{Sc}+$ & $\mathrm{Sc}+$ & $1428+2727$ & Irr & $\mathrm{Sc}+$ \\
\hline $0013+1942$ & $\mathrm{Sc}+$ & $\mathrm{Sc}+$ & $0156+2410$ & $\mathrm{Sb}$ & $\mathrm{Sc}+$ & $1429+2645$ & $\mathrm{Sb}$ & $\mathrm{Sc}+$ \\
\hline $0014+1829$ & $\mathrm{Sa}$ & $\mathrm{Sa}$ & $0157+2413$ & $\mathrm{Sc}+$ & $\mathrm{Sc}+$ & $1430+2947$ & So & S0 \\
\hline $0014+1748$ & $\mathrm{SBb}$ & $\mathrm{SBb}$ & $0157+2102$ & $\mathrm{Sb}$ & $\mathrm{Sb}$ & $1431+2854$ & $\mathrm{Sb}$ & $\mathrm{Sb}$ \\
\hline $0015+2212$ & $\mathrm{Sa}$ & $\mathrm{Sa}$ & $0159+2354$ & $\mathrm{Sb}$ & $\mathrm{Sa}$ & $1431+2702$ & $\mathrm{Sa}$ & $\mathrm{Sb}$ \\
\hline $0017+1942$ & $\mathrm{Sc}+$ & $\mathrm{Sc}+$ & $0159+2326$ & $\mathrm{Sc}+$ & $\mathrm{Sc}+$ & $1431+2947$ & $\mathrm{BCD}$ & $\mathrm{BCD}$ \\
\hline $0017+2148$ & $\mathrm{Sa}$ & - & $1246+2727$ & Irr & - & $1431+2814$ & $\mathrm{Sb}$ & $\mathrm{Sa}$ \\
\hline $0018+2216$ & $\mathrm{Sb}$ & $\mathrm{Sb}$ & $1247+2701$ & $\mathrm{Sc}+$ & $\mathrm{Sc}+$ & $1432+2645$ & $\mathrm{SBb}$ & $\mathrm{SBb}$ \\
\hline $0018+2218$ & $\mathrm{Sb}$ & - & $1248+2912$ & $\mathrm{SBb}$ & - & $1440+2521 \mathrm{~S}$ & $\mathrm{Sb}$ & $\mathrm{Sb}$ \\
\hline $0019+2201$ & $\mathrm{Sb}$ & $\mathrm{Sc}+$ & $1253+2756$ & $\mathrm{Sa}$ & $\mathrm{Sa}$ & $1440+2511$ & $\mathrm{Sb}$ & $\mathrm{Sb}$ \\
\hline $0022+2049$ & $\mathrm{Sb}$ & $\mathrm{Sb}$ & $1254+2741$ & $\mathrm{Sb}$ & $\mathrm{Sb}$ & $1440+2521 \mathrm{~N}$ & $\mathrm{Sb}$ & $\mathrm{Sa}$ \\
\hline $0023+1908$ & $\mathrm{Sc}+$ & - & $1254+2802$ & $\mathrm{Sc}+$ & $\mathrm{Sc}+$ & $1442+2845$ & $\mathrm{Sb}$ & $\mathrm{Sb}$ \\
\hline $0034+2119$ & $\mathrm{SBc}+$ & - & $1255+2819$ & $\mathrm{Sb}$ & $\mathrm{Sb}$ & $1443+2714$ & $\mathrm{Sa}$ & $\mathrm{Sa}$ \\
\hline $0037+2226$ & $\mathrm{SBc}+$ & - & $1255+3125$ & $\mathrm{Sa}$ & $\mathrm{Sa}$ & $1443+2844$ & $\mathrm{SBc}+$ & $\mathrm{SBc}+$ \\
\hline $0038+2259$ & $\mathrm{Sb}$ & $\mathrm{Sa}$ & $1255+2734$ & $\mathrm{Sc}+$ & Irr & $1443+2548$ & $\mathrm{Sc}+$ & $\mathrm{Sc}+$ \\
\hline $0039+0054$ & $\mathrm{Sc}+$ & - & $1256+2717$ & S0 & - & $1444+2923$ & So & S0 \\
\hline $0040+0257$ & $\mathrm{Sb}$ & $\mathrm{Sc}+$ & $1256+2732$ & INTER & - & $1452+2754$ & $\mathrm{Sb}$ & $\mathrm{Sb}$ \\
\hline $0040+2312$ & $\mathrm{Sc}+$ & - & $1256+2701$ & $\mathrm{Sc}+$ & Irr & $1506+1922$ & $\mathrm{Sb}$ & $\mathrm{Sb}$ \\
\hline $0040+0220$ & $\mathrm{Sc}+$ & $\mathrm{Sb}$ & $1256+2910$ & $\mathrm{Sb}$ & $\mathrm{Sb}$ & $1513+2012$ & $\mathrm{Sa}$ & S0 \\
\hline $0040-0023$ & $\mathrm{Sc}+$ & - & $1256+2823$ & $\mathrm{Sb}$ & $\mathrm{Sb}$ & $1537+2506 \mathrm{~N}$ & $\mathrm{SBb}$ & $\mathrm{SBb}$ \\
\hline $0041+0134$ & $\mathrm{Sc}+$ & - & $1256+2754$ & $\mathrm{Sa}$ & $\mathrm{Sa}$ & $1537+2506 \mathrm{~S}$ & $\mathrm{SBa}$ & $\mathrm{SBa}$ \\
\hline $0043+0245$ & $\mathrm{Sc}+$ & - & $1256+2722$ & $\mathrm{Sc}+$ & $\mathrm{Sc}+$ & $1557+1423$ & $\mathrm{Sb}$ & $\mathrm{Sb}$ \\
\hline $0043-0159$ & $\mathrm{Sc}+$ & - & $1257+2808$ & $\mathrm{Sb}$ & $\mathrm{Sa}$ & $1612+1308$ & $\mathrm{BCD}$ & $\mathrm{BCD}$ \\
\hline $0044+2246$ & $\mathrm{Sb}$ & $\mathrm{Sb}$ & $1258+2754$ & $\mathrm{Sb}$ & $\mathrm{Sb}$ & $1646+2725$ & $\mathrm{Sc}+$ & $\mathrm{Sc}+$ \\
\hline $0045+2206$ & INTER & & $1259+2934$ & $\mathrm{Sb}$ & $\mathrm{Sb}$ & $1647+2950$ & $\mathrm{Sc}+$ & $\mathrm{Sc}+$ \\
\hline $0047+2051$ & $\mathrm{Sc}+$ & $\mathrm{Sc}+$ & $1259+3011$ & $\mathrm{Sa}$ & $\mathrm{Sa}$ & $1647+2729$ & $\mathrm{Sb}$ & $\mathrm{Sb}$ \\
\hline $0047-0213$ & S0 & $\mathrm{Sa}$ & $1259+2755$ & $\mathrm{Sa}$ & $\mathrm{Sa}$ & $1647+2727$ & $\mathrm{Sb}$ & $\mathrm{Sa}$ \\
\hline $0047+2413$ & $\mathrm{Sa}$ & $\mathrm{Sa}$ & $1300+2907$ & $\mathrm{Sa}$ & $\mathrm{Sb}$ & $1648+2855$ & $\mathrm{Sa}$ & $\mathrm{Sa}$ \\
\hline $0047+2414$ & $\mathrm{Sc}+$ & - & $1301+2904$ & $\mathrm{Sb}$ & $\mathrm{Sb}$ & $1653+2644$ & INTER & - \\
\hline $0049-0006$ & $\mathrm{BCD}$ & $\mathrm{BCD}$ & $1302+2853$ & $\mathrm{Sb}$ & $\mathrm{Sa}$ & $1654+2812$ & $\mathrm{Sc}+$ & $\mathrm{Sc}+$ \\
\hline $0049+0017$ & $\mathrm{Sb}$ & $\mathrm{Sc}+$ & $1302+3032$ & $\mathrm{Sa}$ & - & $1655+2755$ & $\mathrm{Sc}+$ & $\mathrm{Sb}$ \\
\hline $0049-0045$ & $\mathrm{Sb}$ & - & $1303+2908$ & Irr & Irr & $1656+2744$ & $\mathrm{Sa}$ & $\mathrm{Sa}$ \\
\hline $0050+0005$ & $\mathrm{Sa}$ & $\mathrm{Sa}$ & $1304+2808$ & $\mathrm{Sb}$ & $\mathrm{Sa}$ & $1657+2901$ & $\mathrm{Sb}$ & $\mathrm{Sc}+$ \\
\hline $0050+2114$ & $\mathrm{Sa}$ & $\mathrm{Sa}$ & $1304+2830$ & $\mathrm{BCD}$ & $\mathrm{BCD}$ & $1659+2928$ & SB0 & SB0 \\
\hline $0051+2430$ & $\mathrm{Sa}$ & - & $1304+2907$ & $\operatorname{Irr}$ & Irr & $1701+3131$ & S0 & S0 \\
\hline $0054-0133$ & $\mathrm{Sb}$ & - & $1304+2818$ & $\mathrm{Sc}+$ & $\mathrm{Sc}+$ & $2238+2308$ & $\mathrm{Sa}(\mathrm{r})$ & $\mathrm{Sa}$ \\
\hline $0054+2337$ & $\mathrm{Sc}+$ & - & $1306+2938$ & $\mathrm{SBb}$ & $\mathrm{Sb}$ & $2239+1959$ & S0 & S0 \\
\hline $0056+0044$ & Irr & Irr & $1306+3111$ & $\mathrm{Sc}+$ & $\mathrm{Sc}+$ & $2249+2149$ & $\mathrm{Sb}$ & $\mathrm{Sa}$ \\
\hline $0056+0043$ & $\mathrm{Sb}$ & $\mathrm{Sc}+$ & $1307+2910$ & $\mathrm{SBb}$ & $\mathrm{SBb}$ & $2250+2427$ & $\mathrm{Sa}$ & $\mathrm{Sa}$ \\
\hline $0119+2156$ & $\mathrm{Sb}$ & $\mathrm{Sc}+$ & $1308+2958$ & $\mathrm{Sc}+$ & $\mathrm{Sc}+$ & $2251+2352$ & $\mathrm{Sc}+$ & $\mathrm{Sc}+$ \\
\hline $0121+2137$ & $\mathrm{Sc}+$ & $\mathrm{Sc}+$ & $1308+2950$ & $\mathrm{SBb}$ & $\mathrm{SBb}$ & $2253+2219$ & $\mathrm{Sa}$ & $\mathrm{Sa}$ \\
\hline $0129+2109$ & $\mathrm{SBc}+$ & - & $1310+3027$ & $\mathrm{Sb}$ & $\mathrm{Sa}$ & $2255+1930 \mathrm{~S}$ & $\mathrm{Sb}$ & $\mathrm{Sb}$ \\
\hline $0134+2257$ & $\mathrm{Sb}$ & - & $1312+3040$ & $\mathrm{Sa}$ & $\mathrm{Sa}$ & $2255+1930 \mathrm{~N}$ & $\mathrm{Sb}$ & $\mathrm{Sb}$ \\
\hline $0135+2242$ & S0 & So & $1312+2954$ & $\mathrm{Sc}+$ & $\mathrm{Sc}+$ & $2255+1926$ & $\mathrm{Sb}$ & $\mathrm{Sc}+$ \\
\hline $0138+2216$ & $\mathrm{Sc}+$ & - & $1313+2938$ & $\mathrm{Sa}$ & $\mathrm{Sa}$ & $2255+1654$ & $\mathrm{Sc}+$ & $\mathrm{Sc}+$ \\
\hline
\end{tabular}


Table 5. continued

\begin{tabular}{lcc|lcc|ccc}
\hline UCM name & MpT $(B)$ & MpT $(r)$ & UCM name & MpT $(B)$ & MpT $(r)$ & UCM name & MpT $(B)$ & MpT $(r)$ \\
$(1)$ & $(2)$ & $(3)$ & $(1)$ & $(2)$ & $(3)$ & $(1)$ & $(2)$ & $(3)$ \\
\hline \hline $2256+2001$ & $\mathrm{Sc}+$ & $\mathrm{Sc}+$ & $2313+1841$ & $\mathrm{Sb}$ & $\mathrm{Sb}$ & $2325+2318$ & $\mathrm{INTER}$ & - \\
$2257+2438$ & $\mathrm{~S} 0$ & $\mathrm{~S} 0$ & $2313+2517$ & $\mathrm{Sa}$ & - & $2325+2208$ & $\mathrm{SBc}+$ & $\mathrm{SBc}+$ \\
$2257+1606$ & $\mathrm{~S} 0$ & - & $2315+1923$ & $\mathrm{Sb}$ & $\mathrm{Sa}$ & $2326+2435$ & $\mathrm{Sb}$ & $\mathrm{Sa}$ \\
$2258+1920$ & $\mathrm{Sc}+$ & $\mathrm{Sc}+$ & $2316+2457$ & $\mathrm{SBa}$ & $\mathrm{SBa}$ & $2327+2515 \mathrm{~N}$ & $\mathrm{Sb}$ & $\mathrm{Sb}$ \\
$2300+2015$ & $\mathrm{Sb}$ & $\mathrm{Sb}$ & $2316+2459$ & $\mathrm{Sc}+$ & $\mathrm{Sc}+$ & $2327+2515 \mathrm{~S}$ & $\mathrm{~S} 0$ & $\mathrm{~S} 0$ \\
$2302+2053 \mathrm{~W}$ & $\mathrm{Sb}$ & $\mathrm{Sb}$ & $2316+2028$ & $\mathrm{Sa}$ & $\mathrm{Sc}+$ & $2329+2427$ & $\mathrm{Sb}$ & $\mathrm{Sb}$ \\
$2302+2053 \mathrm{E}$ & $\mathrm{Sb}$ & $\mathrm{Sb}$ & $2317+2356$ & $\mathrm{Sa}$ & $\mathrm{Sa}$ & $2329+2500$ & $\mathrm{~S} 0(\mathrm{r})$ & $\mathrm{S} 0(\mathrm{r})$ \\
$2303+1856$ & $\mathrm{Sa}$ & $\mathrm{Sa}$ & $2319+2234$ & $\mathrm{Sb}$ & $\mathrm{Sc}+$ & $2329+2512$ & $\mathrm{Sa}$ & $\mathrm{Sa}$ \\
$2303+1702$ & $\mathrm{Sc}+$ & $\mathrm{Sc}+$ & $2319+2243$ & $\mathrm{~S} 0$ & $\mathrm{~S} 0$ & $2331+2214$ & $\mathrm{Sb}$ & $\mathrm{Sb}$ \\
$2304+1640$ & $\mathrm{SCD}$ & $\mathrm{BCD}$ & $2320+2428$ & $\mathrm{Sa}$ & $\mathrm{Sa}$ & $2333+2248$ & $\mathrm{Sc}+$ & $\mathrm{Sc}+$ \\
$2304+1621$ & $\mathrm{Sa}$ & $\mathrm{Sa}$ & $2321+2149$ & $\mathrm{Sc}+$ & $\mathrm{Sc}+$ & $2333+2359$ & $\mathrm{~S} 0 \mathrm{a}$ & $\mathrm{S} 0$ \\
$2307+1947$ & $\mathrm{Sb}$ & $\mathrm{Sb}$ & $2321+2506$ & $\mathrm{Sc}+$ & $\mathrm{Sc}+$ & $2348+2407$ & $\mathrm{Sa}$ & $\mathrm{Sa}$ \\
$2310+1800$ & $\mathrm{Sb}$ & $\mathrm{Sc}+$ & $2322+2218$ & $\mathrm{Sc}+$ & $\mathrm{Sc}+$ & $2351+2321$ & $\mathrm{Sb}$ & $\mathrm{Sb}$ \\
$2312+2204$ & $\mathrm{Sa}$ & - & $2324+2448$ & $\mathrm{Sb}$ & $\mathrm{Sc}+$ & & & \\
\hline \hline
\end{tabular}

(1) UCM name. (2) Morphological type established using 5 different criteria based on luminosity concentration and bulge-disk decomposition applied to the Johnson $B$ images. (3) Morphological type established using 5 different criteria based on luminosity concentration and bulge-disk decomposition applied to the Gunn $r$ images.

Table 6. Hubble types for the UCM galaxies

\begin{tabular}{ccccccccc}
\hline Filter & $\mathrm{S} 0$ & $\mathrm{Sa}$ & $\mathrm{Sb}$ & $\mathrm{Sc}+$ & $\mathrm{Irr}$ & $\mathrm{BCD}$ & Int & Total \\
\hline \hline$B$ & 14 & 38 & 69 & 50 & 5 & 8 & 6 & 190 \\
& $7 \%$ & $20 \%$ & $36 \%$ & $26 \%$ & $3 \%$ & $4 \%$ & $3 \%$ & \\
$r$ & 12 & 41 & 43 & 46 & 5 & 8 & - & 155 \\
& $7 \%$ & $27 \%$ & $28 \%$ & $30 \%$ & $3 \%$ & $5 \%$ & & \\
\hline \hline
\end{tabular}

Number of galaxies and percentage of the UCM galaxies according to their Hubble type in the Johnson $B$ and the Gunn $r$ bandpasses.

Table 7. Averaged values of each Hubble type

\begin{tabular}{cccccccc}
\hline Parameter & S0 & Sa & Sb & Sc+ & Irr & BCD & Int \\
\hline \hline$B / T$ & 0.53 & 0.39 & 0.16 & 0.04 & 0.07 & 0.41 & 0.30 \\
\hline$\mu_{\mathrm{e}}$ & 20.6 & 20.7 & 21.3 & 21.5 & 21.8 & 21.5 & 20.1 \\
\hline$c_{31}$ & 5.3 & 3.8 & 3.3 & 2.7 & 2.9 & 3.5 & 5.0 \\
\hline$c_{42}$ & 4.3 & 3.5 & 3.2 & 2.7 & 2.8 & 3.4 & 4.0 \\
\hline$c_{\text {in }}$ & 0.58 & 0.50 & 0.40 & 0.29 & 0.33 & 0.35 & 0.57 \\
\hline$A$ & 0.09 & 0.10 & 0.10 & 0.11 & 0.21 & 0.06 & 0.20 \\
(barred) & 0.06 & 0.15 & 0.11 & 0.12 & - & - & - \\
\hline \hline
\end{tabular}

indices, mean photometric radii and surface brightnesses and asymmetry coefficients.

All the above information has been used to perform the morphological classification of the UCM galaxies. The sample is dominated by late Hubble type objects $(65 \%$ being $\mathrm{Sb}$ or later). We have not found a great difference between this classification and the results achieved with the Gunn $r$ data (Vitores et al. 1996a, 1996b).

Our galaxies are characterized by shorter disks than those of normal spirals. Besides, they seem to be objects with a high luminosity concentration. A preliminary comparison between the characteristics of the sample in the red and blue bandpasses, yields to the result that

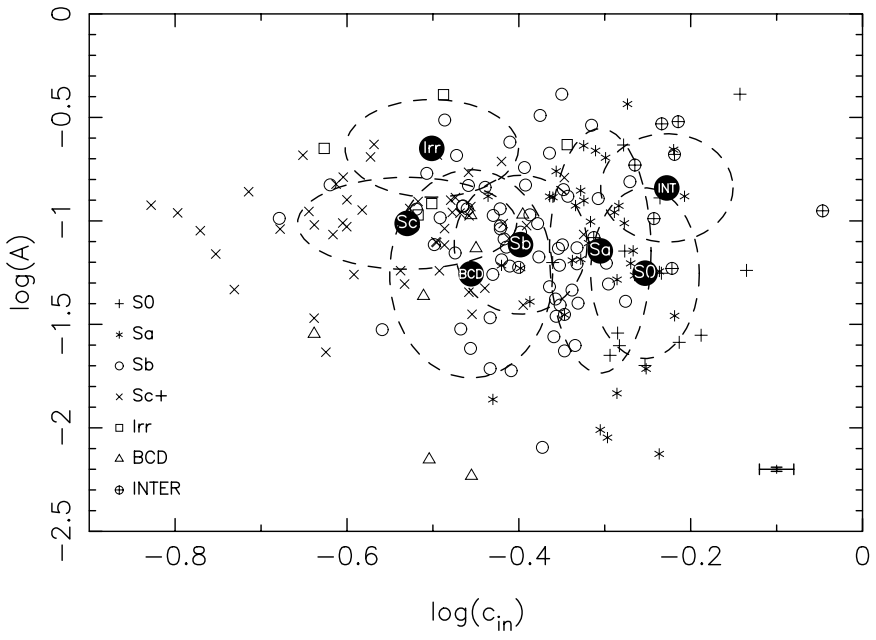

Fig. 17. Concentration index $c_{\text {in }}$ versus asymmetry coefficient. Different symbols stand for distinct morphological types. Ellipses show the median and standard deviation of each parameter for the different morphological types

emission-line galaxies have a higher concentration of blue light than red light in the inner parts of the objects; on the contrary, they also seem to be more extended in $B$ than in $r$.

Finally, a size-luminosity correlation has been outlined. We have also presented several plots where a morphological segregation is patent. These plots involve information about luminosity, concentration of light and asymmetry.

In next papers we will face a most exhaustive comparison between bands, including colour gradients and a stellar population study. Likewise, we will likened our sample to high-redshifts surveys searching for clues to their nature and galaxy evolution. 


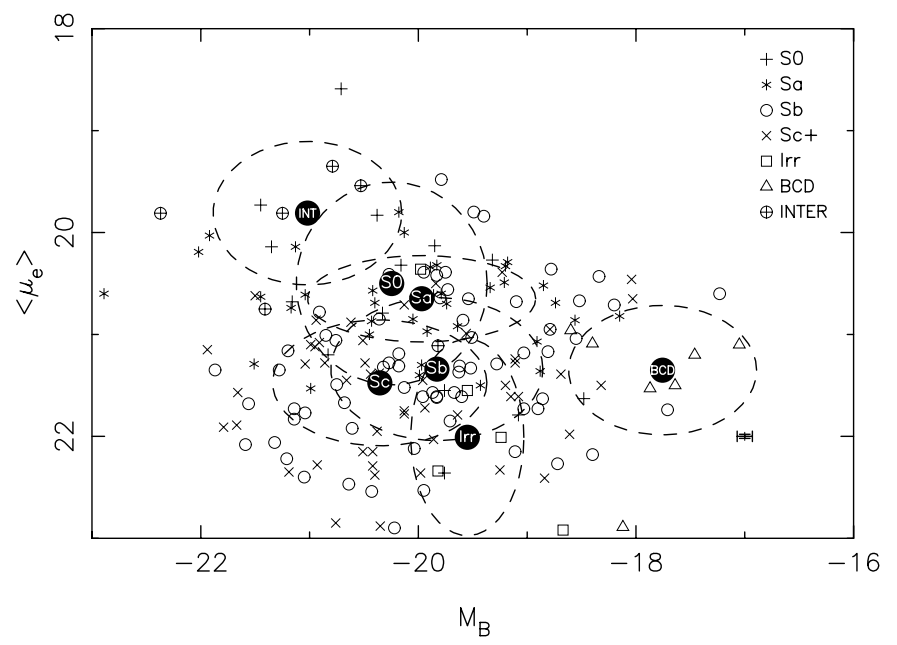

Fig. 18. Mean effective surface brightness versus absolute magnitude. Ellipses show the median and standard deviation of each parameter for the different morphological types

Acknowledgements. This paper is based on observations obtained at the German-Spanish Astronomical Centre, Calar Alto, Spain, operated by the Max-Planck Institute für Astronomie (MPIE), Heidelberg, jointly with the Spanish Commission for Astronomy. It is also partly based on observations made with the Jacobus Kapteyn Telescope operated on the island of La Palma by the Royal Greenwich Observatory in the Spanish Observatorio del Roque de los Muchachos of the Instituto de Astrofísica de Canarias and the $1.52 \mathrm{~m}$ telescope of the EOCA/OAN Observatory.

This research has made use of the NASA/IPAC Extragalactic Database (NED) which is operated by the Jet Propulsion Laboratory, California Institute of Technology, under contract with the National Aeronautics and Space Administration. We have also use of the LEDA database, http://www-obs.univ-lyon1.fr. This research was also supported by the Spanish Programa Sectorial de Promoción General del Conocimiento under grants PB96-0610 and PB960645. P. G. Pérez-González acknowledges the receipt of a "Formación de Profesorado Universitario" fellowship from the Spanish "Ministerio de Educación y Cultura".

We would like to thank C. E. García-Dabó and S. Pascual for their help during part of the observing runs and stimulating conversations. We thank M. N. Estévez for carefully reading the manuscript and making some useful remarks. We are also grateful to Dr. E. Emsellem for his helpful comments and suggestions.

\section{References}

Abraham, R. G., van den Bergh, S., Galzebrook, K., et al. 1996, ApJS, 107, 1

Alonso-Herrero, A., Aragón-Salamanca, A., Zamorano, J., \& Rego, M. 1996, MNRAS, 278, 417

Alonso, O., García-Dabó, E., Zamorano, J., Gallego, J., \& Rego, M. 1999, ApJS, 122, 415

Andredakis, Y. C., \& Sanders, R. H. 1994, MNRAS, 267, 283
Baggett, W. E., Baggett, S. M., \& Anderson, K. S. J. 1998, AJ, 116, 1626

Bershady, M. A., Jangren, A., \& Conselice, C. J. 2000, AJ, 119, 2645

Beijersbergen, M., de Blok, W. J. G., \& van der Hulst, J. M. 1999, A\&A, 351, 903

Bothun, G. D., Halpern, J. P., Lonsdale, C. J., Impey, C., \& Schmitz, M. 1989, ApJS, 70, 271

Boroson, T. 1981, ApJS, 46, 177

Chitre, A., \& Joshi, U. C. 1999, A\&AS, 139, 105

Chatzichristou, E. T. 1999 [astro-ph/9912227]

de Jong, R. S. 1996a, A\&A, 313, 45

de Jong, R. S. 1996b, A\&AS, 118, 557

de Vaucouleurs, G. 1948, Ann. d'Ap., 11, 247

de Vaucouleurs, G. 1977, Evolution of galaxies and stellar populations, ed. R. B. Larson, \& B. M. Tynsley, Yale Univ. Obs., New Haven, 43

Doi, M., Fukugita, M., \& Okamura, S. 1993, MNRAS, 264, 832

Freeman, K. C. 1970, ApJ, 160, 811

Fukugita, M. Shimasaku, K., \& Ichikawa, T. 1995, PASP, 107, 945

Gallego, J., Zamorano, J., Aragón-Salamanca, A., \& Rego, M. 1995, ApJ, 455, L1

Gallego, J. 1999, Ap\&SS, 263, 1

Gallego, J., Zamorano, J., Rego, M., Alonso, O., \& Vitores, A. G. 1996, A\&AS, 120, 323

Gallego, J., Zamorano, J., Rego, M., Alonso, O., \& Vitores, A. G. 1997, ApJ, 475, 502

Gavazzi, G., Garilli, B., \& Boselli, A. 1990, A\&AS, 83, 399

Gil de Paz, A., Aragón-Salamanca, A., Gallego, J., et al. 2000, MNRAS, 316, 357

Hu, E. M., Cowie, L. L., \& McMahon, R. G. 1998, ApJ, 502, 99

Hunt, L. K., \& Malkan, M. A. 1999, ApJ, 516, 660

Hunt, L. K., Malkan, M. A., Moriondo, G., \& Salvati, M. 1999, ApJ, 510, 637

Jedrzejewski, P. 1987, MNRAS, 226, 747

Knapen, J. H., \& van der Kruit, P. C. 1991, A\&A, 248, 57

Kent, S. M. 1985, ApJS, 59, 115

Kron, R. G. 1980, ApJS, 43, 305

McGaugh, S. S., Bothun, G. D., \& Schombert, J. M. 1995, AJ, 110,573

Pérez-González, P. G., Zamorano, J., Gallego, J., \& Gil de Paz, A. 2000, A\&AS, 141, 409

Pritchet, C., \& Kline, M. I. 1981, AJ, 86, 1859

Schombert, J. M., \& Bothun, G. D. 1987, AJ, 93, 60

Simien, F. 1989, Photometric decomposition of galaxies, in The World of Galaxies, ed. H. G. Corwin, \& L. Bottinelli (Springer-Verlag, New York), 293

Simien, F., \& de Vaucouleurs, G. 1986, ApJ, 282, 95

Steidel, C. C., Adelberger, K. L., Giavalisco, M., Dickinson, M., \& Pettini, M. 1999, ApJ, 519, 1

Vennik, J., Hopp, U., \& Popescu, C. C. 2000, A\&AS, 142, 399

Vitores, A. G., Zamorano, J., Rego, M., Alonso, O., \& Gallego, J. 1996a, A\&AS, 118, 7

Vitores, A. G., Zamorano, J., Rego, M., Gallego, J., \& Alonso, O. 1996b, A\&AS, 120, 385

Zamorano, J., Rego, M., Gallego, J., et al. 1994, ApJS, 95, 387

Zamorano, J., Gallego, J., Rego, M., Vitores, A. G., \& Alonso, O. 1996, ApJS, 105, 343 